NISSUNA UMANA INVESTIGAZIONE SI PUO DIMANDARE VERA SCIENZIA S'ESSA NON PASSA PER LE MATEMATICHE DIMOSTRAZIONI LEONARDO DA VINCI

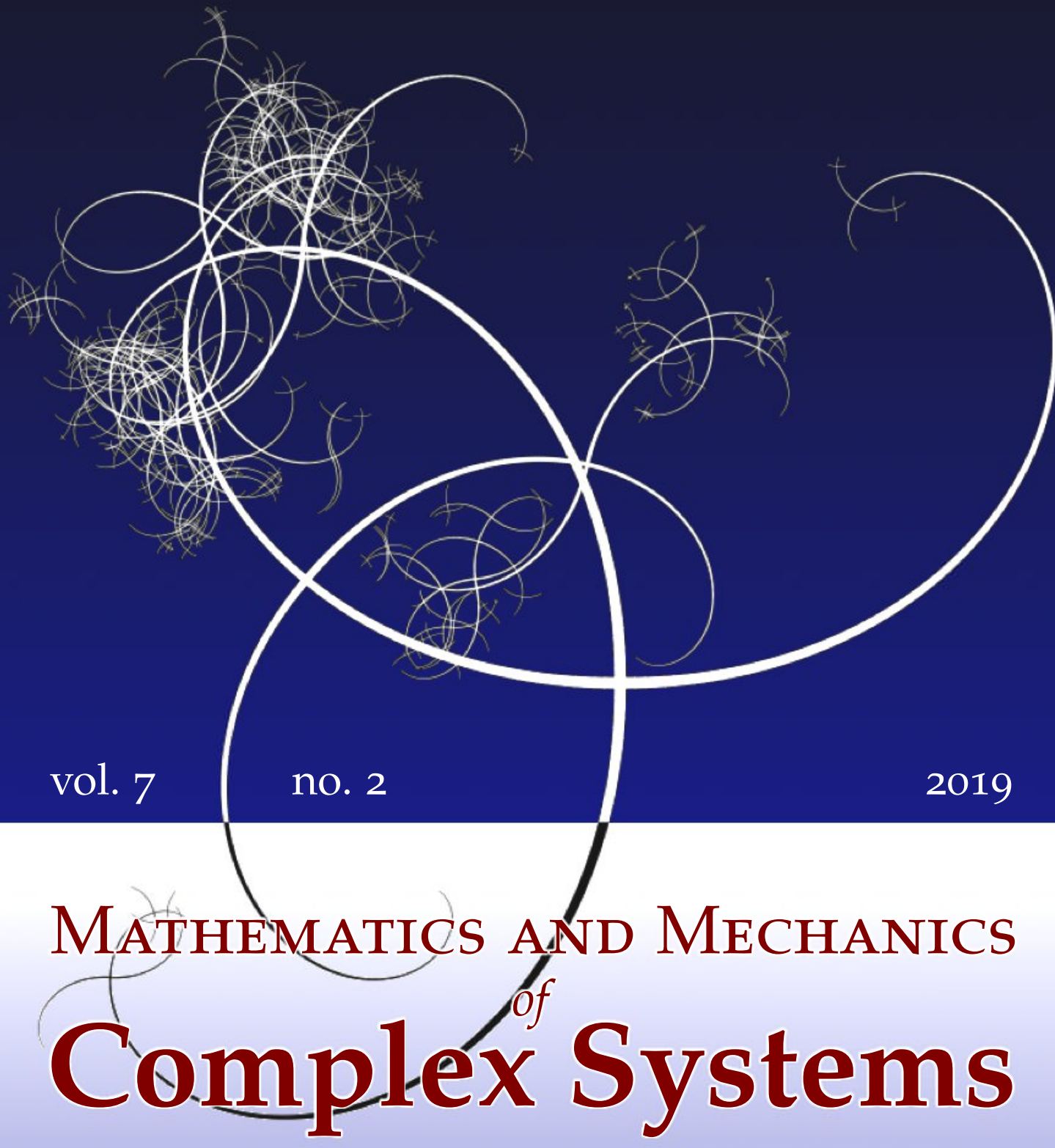

SERGEy GAVRILyUK AND HenRi GOUIN

DYNAMIC BOUNDARY CONDITIONS

FOR MEMBRANES WHOSE SURFACE ENERGY DEPENDS ON THE MEAN AND GAUSSIAN CURVATURES 


\title{
DYNAMIC BOUNDARY CONDITIONS FOR MEMBRANES WHOSE SURFACE ENERGY DEPENDS ON THE MEAN AND GAUSSIAN CURVATURES
}

\author{
SERgey GAVRILyuk AND Henri Gouin
}

\begin{abstract}
Membranes are an important subject of study in physical chemistry and biology. They can be considered as material surfaces with a surface energy depending on the curvature tensor. Usually, mathematical models developed in the literature consider the dependence of surface energy only on mean curvature with an added linear term for Gauss curvature. Therefore, for closed surfaces the Gauss curvature term can be eliminated because of the Gauss-Bonnet theorem. Rosso and Virga (Proc. Roy. Soc. Lond. A 455:1992 (1999), 4145-4168) considered the dependence on the mean and Gaussian curvatures in statics and under a restrictive assumption of the membrane inextensibility. The authors derived the shape equation as well as two scalar boundary conditions on the contact line.

In this paper - thanks to the principle of virtual working - the equations of motion and boundary conditions governing the fluid membranes subject to general dynamical bending are derived without the membrane inextensibility assumption. We obtain the dynamic "shape equation" (equation for the membrane surface) and the dynamic conditions on the contact line generalizing the classical Young-Dupré condition.
\end{abstract}

\section{Introduction}

The study of equilibrium, for small wetting droplets placed on a curved rigid surface, is an old problem of continuum mechanics. When the droplets' size is of micron range the droplet volume energy can be neglected. The surface energy of the surface $S$ can be expressed in the form

$$
E=\iint_{S} \sigma d s
$$

where $\sigma$ denotes the energy per unit surface. Two types of surfaces are present in physical problems:

\section{Communicated by Pierre Seppecher.}

PACS2010: 45.20.dg, 68.03.Cd, 68.35.Gy, 02.30.Xx.

MSC2010: primary 74K15, 76Z99; secondary 92C37.

Keywords: surface energy, curvature tensor, dynamic boundary conditions. 
- rigid surfaces (only the kinematic boundary condition is imposed) and

- free surfaces (both the kinematic and dynamic boundary conditions are imposed).

We will see the difference between the energy variation in the case of rigid and free surfaces.

The simplest case corresponds to a constant surface energy $\sigma$, but in general, $\sigma$ also depends on physical parameters (temperature, surfactant concentrations, etc. [Gouin 2014a; Rocard 1952; Steigmann and Li 1995]) and geometrical parameters (invariants of curvature tensor). The last case is important in biology and, in particular, in the dynamics of vesicles [Alberts et al. 2002; Lipowsky and Sackmann 1995; Seifert 1997]. Vesicles are small liquid droplets with a diameter of a few tens of micrometers, bounded by an impermeable lipid membrane of a few nanometers thick. The membranes are homogeneous down to molecular dimensions. Consequently, it is possible to model the boundary of vesicle as a two-dimensional smooth surface whose energy per unit surface $\sigma$ is a function both of the sum (denoted by $H$ ) and product (denoted by $K$ ) of principal curvatures of the curvature tensor:

$$
\sigma=\sigma(H, K) .
$$

In mathematical description of biological membranes, one often uses the Helfrich energy [1973; Tu 2011]:

$$
\sigma(H, K)=\sigma_{0}+\frac{\kappa}{2}\left(H-H_{0}\right)^{2}+\bar{\kappa} K,
$$

where $\sigma_{0}, H_{0}, \kappa$, and $\bar{\kappa}$ are dimensional constants. Another purely mathematical example is the Wilmore energy [1993]:

$$
\sigma(H, K)=H^{2}-4 K .
$$

This energy measures the "roundness" of the free surface. For a given volume, this energy is minimal in case of spheres. One can also propose another surface energy in the form

$$
\sigma=\sigma_{0}+h_{0}\left(H^{2}-H_{0}^{2}\right)^{2}+k_{0}\left(K-K_{0}\right)^{2},
$$

where $\sigma_{0}, h_{0}, H_{0}, k_{0}$, and $K_{0}$ are dimensional constants. This kind of energy is invariant under the change of sign of principal curvatures, (i.e., the change of sign yields $H \rightarrow-H$ and $K \rightarrow K$ ). It can thus describe the "mirror buckling" phenomenon: a portion of the membrane inverts to form a cap with equal but opposite principal curvatures. It is also a homogeneous function of degree four with respect to principal curvatures.

The equilibrium for membranes (called "shape equation" by Helfrich) is formulated in numerous papers and references herein [Biscari et al. 2004; Capovilla and 
Guven 2002; Fournier 2007; Helfrich 1973; Napoli and Vergori 2010; Zhong-can and Helfrich 1989]. The "edge conditions" (boundary conditions at the contact line) are formulated in few papers and only in statics. In particular, in [Rosso and Virga 1999] the shape equation and two boundary conditions are formulated for the general dependence $\sigma(H, K)$ under the assumption of the membrane inextensibility. However, the boundary conditions obtained do not contain the classical Young-Dupré condition for the constant surface energy. In the case where the energy depends only on $H$ a static generalization of Young-Dupré condition was derived in [Gouin 2014b].

The aim of our paper is to develop the theory of moving membranes which are in contact with a solid surface. The surface energy of the membrane will be a function both of $H$ and $K$. We obtain a set of boundary conditions on the moving interfaces (membranes) as well as on the moving edges.

The motion of a continuous medium is represented by a diffeomorphism $\phi$ of a three-dimensional reference configuration $D_{0}$ into the physical space. In order to analytically describe the transformation, variables $X=\left(X^{1}, X^{2}, X^{3}\right)^{T}$ single out individual particles corresponding to material or Lagrangian coordinates, where superscript " $T$ " means the transposition. The transformation representing the motion of a continuous medium occupying the material volume $D_{t}$ is

$$
\boldsymbol{x}=\boldsymbol{\phi}(t, \boldsymbol{X}) \quad \text { or } \quad x^{i}=\phi^{i}\left(t, X^{1}, X^{2}, X^{3}\right), \quad i=\{1,2,3\},
$$

where $t$ denotes the time and $\boldsymbol{x}=\left(x^{1}, x^{2}, x^{3}\right)^{T}$ denote the Eulerian coordinates. At $t$ fixed, the transformation possesses an inverse and has continuous derivatives up to the second order (the dependence of the surface energy on the curvature tensor will regularize the solutions, so the cusps and shocks do not appear).

At equilibrium, the unit normal vector to a static surface $\varphi_{0}(\boldsymbol{x})=0$ is the gradient of the so-called signed distance function defined as follows. Let

$$
d(\boldsymbol{x})=\left\{\begin{array}{cl}
\min |\boldsymbol{x}-\boldsymbol{\xi}| & \text { if } \varphi_{0}>0 \\
0 & \text { if } \varphi_{0}=0 \\
-\min |\boldsymbol{x}-\boldsymbol{\xi}| & \text { if } \varphi_{0}<0
\end{array}\right.
$$

where the minimum is taken over points $\xi$ at the surface, and $|\cdot|$ denotes the Euclidean norm. The unit normal vector is

$$
\boldsymbol{n}=\nabla d(\boldsymbol{x}) .
$$

In dynamical problems, the main difficulty in formulating boundary conditions comes from the fact that one cannot assume that for all time $t$ the unit normal vector to the surface is the gradient of the signed distance function.

Indeed, if the material surface is moving, i.e., the surface position depends on time $t$, the surface points of the continuum medium are also moving and they will 
depend implicitly on $\boldsymbol{x}$. Let $\varphi(t, \boldsymbol{x})=0$ be the position of the material surface at time $t$. Its evolution is determined by the equation

$$
\varphi_{t}+\boldsymbol{u}^{T} \nabla \varphi=0
$$

where $\boldsymbol{u}$ is the velocity of particles at the surface. Equation (3) is the classical kinematic condition for material moving interfaces. Let us derive the equation for the norm of $\nabla \varphi$. Taking the gradient of (3) and multiplying by $\nabla \varphi$, one obtains

$$
(|\nabla \varphi|)_{t}+\boldsymbol{n}^{T} \nabla\left(\boldsymbol{u}^{T} \nabla \varphi\right)=0,
$$

where $\boldsymbol{n}=\nabla \varphi /|\nabla \varphi|$ is the unit normal vector to surface $\varphi(t, \boldsymbol{x})=0$. It follows from (4) that, even if initially $|\nabla \varphi|=1$ (i.e., unit normal $\boldsymbol{n}$ is defined at $t=0$ as the gradient of the signed distance function), this property is not conserved in time.

The following definitions and notations are used in the paper. For any vectors $\boldsymbol{a}, \boldsymbol{b}$, we write $\boldsymbol{a}^{T} \boldsymbol{b}$ for their scalar product (the line vector is multiplied by the column vector), and $\boldsymbol{a} \boldsymbol{b}^{T}$ for their tensor product (the column vector is multiplied by the line vector). The last product is usually denoted as $\boldsymbol{a} \otimes \boldsymbol{b}$. The product of a second-order tensor $\boldsymbol{A}$ by a vector $\boldsymbol{a}$ is denoted by $\boldsymbol{A} \boldsymbol{a}$. Notation $\boldsymbol{b}^{T} \boldsymbol{A}$ means the covector $\boldsymbol{c}^{T}$ defined by the rule $\boldsymbol{c}^{T}=\left(\boldsymbol{A}^{T} \boldsymbol{b}\right)^{T}$. The identity tensor is denoted by $\boldsymbol{I}$.

The divergence of $\boldsymbol{A}$ is covector $\operatorname{div} \boldsymbol{A}$ such that, for any constant vector $\boldsymbol{h}$, one has

$$
(\operatorname{div} \boldsymbol{A}) \boldsymbol{h}=\operatorname{div}(\boldsymbol{A} \boldsymbol{h}),
$$

i.e., the divergence of $\boldsymbol{A}$ is a row vector, in which each component is the divergence of the corresponding column of $\boldsymbol{A}$. It implies

$$
\operatorname{div}(\boldsymbol{A} \boldsymbol{v})=(\operatorname{div} \boldsymbol{A}) \boldsymbol{v}+\operatorname{tr}\left(\boldsymbol{A} \frac{\partial \boldsymbol{v}}{\partial \boldsymbol{x}}\right)
$$

for any vector field $\boldsymbol{v}$. Here tr is the trace operator. If $f$ is a real scalar function of $\boldsymbol{x}, \frac{\partial f}{\partial \boldsymbol{x}}$ is the linear form (line vector) associated with the gradient of $f$ (column vector): $\frac{\partial f}{\partial x}=(\nabla f)^{T}$.

If $\boldsymbol{n}$ is the unit normal vector to a surface, $\boldsymbol{P}=\boldsymbol{I}-\boldsymbol{n} \boldsymbol{n}^{T}$ is the projector on the surface with the classical properties

$$
\boldsymbol{P}^{2}=\boldsymbol{P}, \quad \boldsymbol{P}^{T}=\boldsymbol{P}, \quad \boldsymbol{P n}=\mathbf{0}, \quad \boldsymbol{n}^{T} \boldsymbol{P}=\mathbf{0} .
$$

For any scalar field $f$, the vector field $\boldsymbol{v}$, and second-order tensor field $\boldsymbol{A}$, the tangential surface gradient, tangential surface divergence, Beltrami-Laplace operator, and tangent tensors are defined as

$$
\begin{gathered}
\boldsymbol{v}_{\mathrm{tg}}=\boldsymbol{P} \boldsymbol{v}, \quad \boldsymbol{A}_{\mathrm{tg}}=\boldsymbol{P} \boldsymbol{A}, \quad \nabla_{\mathrm{tg}} f=\boldsymbol{P} \nabla f, \\
\operatorname{div}_{\mathrm{tg}} \boldsymbol{v}_{\mathrm{tg}}=\operatorname{tr}\left(\boldsymbol{P} \frac{\partial \boldsymbol{v}_{\mathrm{tg}}}{\partial \boldsymbol{x}}\right), \quad \Delta_{\mathrm{tg}} f=\operatorname{div}_{\mathrm{tg}}\left(\nabla_{\mathrm{tg}} f\right),
\end{gathered}
$$


and for any constant vector $\boldsymbol{h}$,

$$
\operatorname{div}_{\mathrm{tg}}\left(\boldsymbol{A}_{\mathrm{tg}} \boldsymbol{h}\right)=\operatorname{div}_{\mathrm{tg}}\left(\boldsymbol{A}_{\mathrm{tg}}\right) \boldsymbol{h} .
$$

The following relations between surface operators and classical operators applied to tangential tensors in the sense of previous definitions are valid:

$$
\begin{aligned}
\operatorname{div}_{\mathrm{tg}} \boldsymbol{v}_{\mathrm{tg}} & =\operatorname{div} \boldsymbol{v}_{\mathrm{tg}}+\boldsymbol{n}^{T}\left(\frac{\partial \boldsymbol{n}}{\partial \boldsymbol{x}}\right)^{T} \boldsymbol{v}_{\mathrm{tg}}, \\
\operatorname{div}_{\mathrm{tg}} \boldsymbol{v}_{\mathrm{tg}} & =\boldsymbol{n}^{T} \operatorname{rot}\left(\boldsymbol{n} \times \boldsymbol{v}_{\mathrm{tg}}\right), \\
\operatorname{div}_{\mathrm{tg}} \boldsymbol{A}_{\mathrm{tg}} & =\operatorname{div} \boldsymbol{A}_{\mathrm{tg}}+\boldsymbol{n}^{T}\left(\frac{\partial \boldsymbol{n}}{\partial \boldsymbol{x}}\right)^{T} \boldsymbol{A}_{\mathrm{tg}}, \\
\operatorname{div}_{\mathrm{tg}}\left(f \boldsymbol{v}_{\mathrm{tg}}\right) & =f \operatorname{div}_{\mathrm{tg}} \boldsymbol{v}_{\mathrm{tg}}+\left(\nabla_{\mathrm{tg}} f\right)^{T} \boldsymbol{v}_{\mathrm{tg}}, \\
\operatorname{div}_{\mathrm{tg}}\left(f \boldsymbol{A}_{\mathrm{tg}}\right) & =f \operatorname{div}_{\mathrm{tg}} \boldsymbol{A}_{\mathrm{tg}}+\left(\nabla_{\mathrm{tg}} f\right)^{T} \boldsymbol{A}_{\mathrm{tg}},
\end{aligned}
$$

where rot denotes the curl operator. The proof is straightforward. Indeed, since

$$
\frac{\partial\left(\boldsymbol{n}^{T} \boldsymbol{v}_{\mathrm{tg}}\right)}{\partial \boldsymbol{x}}=\boldsymbol{n}^{T}\left(\frac{\partial \boldsymbol{v}_{\mathrm{tg}}}{\partial \boldsymbol{x}}\right)+\boldsymbol{v}_{\mathrm{tg}}^{T}\left(\frac{\partial \boldsymbol{n}}{\partial \boldsymbol{x}}\right)=0
$$

one has

$$
\operatorname{div}_{\mathrm{tg}} \boldsymbol{v}_{\mathrm{tg}}=\operatorname{tr}\left(\boldsymbol{P} \frac{\partial \boldsymbol{v}_{\mathrm{tg}}}{\partial \boldsymbol{x}}\right)=\operatorname{div} \boldsymbol{v}_{\mathrm{tg}}-\boldsymbol{n}^{T}\left(\frac{\partial \boldsymbol{v}_{\mathrm{tg}}}{\partial \boldsymbol{x}}\right) \boldsymbol{n}=\operatorname{div} \boldsymbol{v}_{\mathrm{tg}}+\boldsymbol{n}^{T}\left(\frac{\partial \boldsymbol{n}}{\partial \boldsymbol{x}}\right)^{T} \boldsymbol{v}_{\mathrm{tg}}
$$

which proves relation (5). To prove relation (6), one uses the following identity valid for any vector fields $\boldsymbol{a}$ and $\boldsymbol{b}$ :

$$
\operatorname{rot}(\boldsymbol{a} \times \boldsymbol{b})=\boldsymbol{a} \operatorname{div} \boldsymbol{b}-\boldsymbol{b} \operatorname{div} \boldsymbol{a}+\frac{\partial \boldsymbol{a}}{\partial \boldsymbol{x}} \boldsymbol{b}-\frac{\partial \boldsymbol{b}}{\partial \boldsymbol{x}} \boldsymbol{a} .
$$

We apply this identity to the vectors $\boldsymbol{a}=\boldsymbol{n}$ and $\boldsymbol{b}=\boldsymbol{v}_{\mathrm{tg}}$. Multiplying on the left by $\boldsymbol{n}^{T}$, one obtains relation (6). Relations (7), (8), and (9) are direct consequences of relation (5).

\section{Curvature tensor}

The unit normal vector being prolonged in the surface vicinity, we can directly obtain the expression of its derivative:

$$
\frac{\partial \boldsymbol{n}}{\partial \boldsymbol{x}}=\boldsymbol{P} \frac{\varphi^{\prime \prime}}{|\nabla \varphi|},
$$

where $\varphi^{\prime \prime}$ is the Hessian matrix of $\varphi$ with respect to $\boldsymbol{x}$. One obviously has

$$
\boldsymbol{n}^{T} \frac{\partial \boldsymbol{n}}{\partial \boldsymbol{x}}=\mathbf{0} .
$$


However, since in dynamics $\boldsymbol{n}$ is not the gradient of the signed distance function, we cannot have the property

$$
\frac{\partial \boldsymbol{n}}{\partial \boldsymbol{x}} \boldsymbol{n}=\mathbf{0} .
$$

The curvature tensor is defined as

$$
\boldsymbol{R}=-\boldsymbol{P} \frac{\varphi^{\prime \prime}}{|\nabla \varphi|} \boldsymbol{P}=-\frac{\partial \boldsymbol{n}}{\partial \boldsymbol{x}} \boldsymbol{P} .
$$

Hence, in dynamics

$$
\boldsymbol{R} \neq-\frac{\partial \boldsymbol{n}}{\partial \boldsymbol{x}}
$$

Let us note that the derivation of the shape equation and boundary conditions in statics always uses property (10) and the curvature tensor coming from the definition of the signed distance function. In dynamics, we cannot use these properties and new tools should be developed.

Tensor $\boldsymbol{R}$ is symmetric and has zero as an eigenvalue:

$$
\boldsymbol{R}=\boldsymbol{R}^{T}, \quad \boldsymbol{R} n=\mathbf{0} .
$$

In the eigenbasis, tensor $\boldsymbol{R}$ is diagonal:

$$
\boldsymbol{R}=\left(\begin{array}{ccc}
c_{1} & 0 & 0 \\
0 & c_{2} & 0 \\
0 & 0 & 0
\end{array}\right)
$$

where $c_{1}, c_{2}$ are the principal curvatures. The two invariants of curvature tensor $\boldsymbol{R}$ are

$$
H=c_{1}+c_{2}, \quad K=c_{1} c_{2} .
$$

Invariant $H$ is the double mean curvature, and invariant $K$ is the Gaussian curvature. They can also be expressed in the form

$$
\begin{aligned}
H & =\operatorname{tr} \boldsymbol{R}=-\operatorname{tr}\left(\frac{\partial \boldsymbol{n}}{\partial \boldsymbol{x}}\right) \\
2 K & =(\operatorname{tr} \boldsymbol{R})^{2}-\operatorname{tr}\left(\boldsymbol{R}^{2}\right)=\left[\operatorname{tr}\left(\frac{\partial \boldsymbol{n}}{\partial \boldsymbol{x}}\right)\right]^{2}-\operatorname{tr}\left[\left(\frac{\partial \boldsymbol{n}}{\partial \boldsymbol{x}}\right)^{2}\right]
\end{aligned}
$$

Lemma 1. The following identities are valid:

$$
\begin{aligned}
\operatorname{div}_{\mathrm{tg}} \boldsymbol{P} & =H \boldsymbol{n}^{T}, \\
\operatorname{div}_{\mathrm{tg}} \boldsymbol{R} & =\nabla_{\mathrm{tg}}^{T} H+\left(H^{2}-2 K\right) \boldsymbol{n}^{T}, \\
\boldsymbol{R}^{2} & =H \boldsymbol{R}-K \boldsymbol{P} .
\end{aligned}
$$


Proof. First, let us remark that $\boldsymbol{P}=\boldsymbol{P}_{\mathrm{tg}}$ and $\boldsymbol{R}=\boldsymbol{R}_{\mathrm{tg}}$. One can apply (7) to obtain

$$
\begin{aligned}
\operatorname{div}_{\mathrm{tg}} \boldsymbol{P} & =-\operatorname{div}\left(\boldsymbol{n} \boldsymbol{n}^{T}\right)+\boldsymbol{n}^{T}\left(\frac{\partial \boldsymbol{n}}{\partial \boldsymbol{x}}\right)^{T} \boldsymbol{P} \\
& =-(\operatorname{div} \boldsymbol{n}) \boldsymbol{n}^{T}-\boldsymbol{n}^{T}\left(\frac{\partial \boldsymbol{n}}{\partial \boldsymbol{x}}\right)^{T}+\boldsymbol{n}^{T}\left(\frac{\partial \boldsymbol{n}}{\partial \boldsymbol{x}}\right)^{T}\left(\boldsymbol{I}-\boldsymbol{n} \boldsymbol{n}^{T}\right) \\
& =-(\operatorname{div} \boldsymbol{n}) \boldsymbol{n}^{T},
\end{aligned}
$$

which proves the first relation. The proof of the second relation is as follows:

$$
\begin{aligned}
\operatorname{div} \boldsymbol{R} & =-\operatorname{div}\left(\frac{\partial \boldsymbol{n}}{\partial \boldsymbol{x}}\right)+\operatorname{div}\left(\frac{\partial \boldsymbol{n}}{\partial \boldsymbol{x}} \boldsymbol{n} \boldsymbol{n}^{T}\right) \\
& =-\frac{\partial(\operatorname{div} \boldsymbol{n})}{\partial \boldsymbol{x}}+\operatorname{div}\left(\frac{\partial \boldsymbol{n}}{\partial \boldsymbol{x}} \boldsymbol{n}\right) \boldsymbol{n}^{T}+\boldsymbol{n}^{T}\left(\left(\frac{\partial \boldsymbol{n}}{\partial \boldsymbol{x}}\right)^{2}\right)^{T} \\
& =-\frac{\partial(\operatorname{div} \boldsymbol{n})}{\partial \boldsymbol{x}}+\operatorname{div}\left(\frac{\partial \boldsymbol{n}}{\partial \boldsymbol{x}}\right) \boldsymbol{n} \boldsymbol{n}^{T}+\operatorname{tr}\left(\left(\frac{\partial \boldsymbol{n}}{\partial \boldsymbol{x}}\right)^{2}\right) \boldsymbol{n}^{T}+\boldsymbol{n}^{T}\left(\left(\frac{\partial \boldsymbol{n}}{\partial \boldsymbol{x}}\right)^{2}\right)^{T} \\
& =\frac{\partial H}{\partial \boldsymbol{x}} \boldsymbol{P}+\operatorname{tr}\left(\left(\frac{\partial \boldsymbol{n}}{\partial \boldsymbol{x}}\right)^{2}\right) \boldsymbol{n}^{T}-\boldsymbol{n}^{T}\left(\frac{\partial \boldsymbol{n}}{\partial \boldsymbol{x}}\right)^{T} \boldsymbol{R} .
\end{aligned}
$$

Consequently,

$$
\operatorname{div}_{\mathrm{tg}} \boldsymbol{R}=\frac{\partial H}{\partial \boldsymbol{x}} \boldsymbol{P}+\operatorname{tr}\left(\left(\frac{\partial \boldsymbol{n}}{\partial \boldsymbol{x}}\right)^{2}\right) \boldsymbol{n}^{T}
$$

Using $\operatorname{tr}\left(\left(\frac{\partial \boldsymbol{n}}{\partial \boldsymbol{x}}\right)^{2}\right)=\operatorname{tr}\left(\boldsymbol{R}^{2}\right)=H^{2}-2 K$, we obtain the second relation of the lemma.

Now, the curvature tensor satisfies the Cayley-Hamilton theorem:

$$
\boldsymbol{R}^{3}-H \boldsymbol{R}^{2}+K \boldsymbol{R}=0
$$

The minimal polynomial is

$$
\boldsymbol{R}^{2}-H \boldsymbol{R}+K \boldsymbol{P}=0
$$

which proves the third relation.

\section{Virtual motion}

Define a one-parameter family of virtual motions

$$
\boldsymbol{x}=\boldsymbol{\Phi}(t, \boldsymbol{X}, \lambda)
$$

with scalar $\lambda \in O$, where $O$ is an open real interval containing zero and such that $\boldsymbol{\Phi}(t, \boldsymbol{X}, 0)=\boldsymbol{\phi}(t, \boldsymbol{X})$ (the motion of the continuous medium is obtained for $\lambda=0$ ). 
The virtual displacement of particle $\boldsymbol{X}$ is defined as [Gavrilyuk 2011; Serrin 1959]

$$
\delta \boldsymbol{x}(t, \boldsymbol{X})=\left.\frac{\partial \boldsymbol{\Phi}(t, \boldsymbol{X}, \lambda)}{\partial \lambda}\right|_{\lambda=0} .
$$

In the following, the symbol $\delta$ means the derivative with respect to $\lambda$ at fixed Lagrangian coordinates $\boldsymbol{X}$ and $t$, for $\lambda=0$. We will also denote by $\zeta(t, \boldsymbol{x})$ the virtual displacement expressed as a function of Eulerian coordinates:

$$
\zeta(t, \boldsymbol{x})=\zeta(t, \boldsymbol{\phi}(t, \boldsymbol{X}))=\delta \boldsymbol{x}(t, \boldsymbol{X}) .
$$

\section{Variational tools}

We assume that $D_{t}$ has a smooth boundary $S_{t}$ with edge $C_{t}$. We respectively denote by $D_{0}, S_{0}$, and $C_{0}$ the images of $D_{t}, S_{t}$, and $C_{t}$ in the reference space (of Lagrangian coordinates). The unit vector $\boldsymbol{n}$ and its image $\boldsymbol{n}_{0}$ are the oriented normal vectors to $S_{t}$ and $S_{0}$; the vector $\boldsymbol{t}$ is the oriented unit tangent vector to $C_{t}$ and $\boldsymbol{n}^{\prime}=\boldsymbol{t} \times \boldsymbol{n}$ is the unit binormal vector (see Figure 1). $\boldsymbol{F}=\frac{\partial \phi(t, \boldsymbol{X})}{\partial \boldsymbol{X}} \equiv \frac{\partial \boldsymbol{x}}{\partial \boldsymbol{X}}$ is the deformation gradient. For the sake of simplicity, we will use the same notations for quantities as $\boldsymbol{F}, \boldsymbol{n}$, etc., both in Eulerian and Lagrangian coordinates.

Lemma 2. We have the relations

$$
\begin{aligned}
\delta \operatorname{det} \boldsymbol{F} & =\operatorname{det} \boldsymbol{F} \operatorname{div} \zeta \\
\delta \boldsymbol{n} & =-\boldsymbol{P}\left(\frac{\partial \zeta}{\partial \boldsymbol{x}}\right)^{T} \boldsymbol{n}, \\
\delta\left(\boldsymbol{F}^{-1} \boldsymbol{n}\right) & =-\boldsymbol{F}^{-1} \frac{\partial \zeta}{\partial \boldsymbol{x}} \boldsymbol{n}+\boldsymbol{F}^{-1} \delta \boldsymbol{n}, \\
\delta\left(\frac{\partial \boldsymbol{n}}{\partial \boldsymbol{x}}\right) & =\frac{\partial \delta \boldsymbol{n}}{\partial \boldsymbol{x}}-\frac{\partial \boldsymbol{n}}{\partial \boldsymbol{x}} \frac{\partial \zeta}{\partial \boldsymbol{x}} .
\end{aligned}
$$

Proof of relation (11). The Jacobi formula for the determinant is

$$
\delta(\operatorname{det} \boldsymbol{F})=\operatorname{det} \boldsymbol{F} \operatorname{tr}\left(\boldsymbol{F}^{-1} \delta \boldsymbol{F}\right) .
$$

Also,

$$
\delta \boldsymbol{F}=\delta\left(\frac{\partial \boldsymbol{x}}{\partial \boldsymbol{X}}\right)=\frac{\partial \delta \boldsymbol{x}}{\partial \boldsymbol{X}}
$$

Then

$$
\operatorname{tr}\left(\boldsymbol{F}^{-1} \delta \boldsymbol{F}\right)=\operatorname{tr}\left(\frac{\partial \boldsymbol{X}}{\partial \boldsymbol{x}} \frac{\partial \delta \boldsymbol{x}}{\partial \boldsymbol{X}}\right)=\operatorname{tr}\left(\frac{\partial \delta \boldsymbol{x}}{\partial \boldsymbol{X}} \frac{\partial \boldsymbol{X}}{\partial \boldsymbol{x}}\right)=\operatorname{tr}\left(\frac{\partial \zeta}{\partial \boldsymbol{x}}\right)=\operatorname{div} \zeta .
$$

Proof of relation (12). Surface $\varphi(t, \boldsymbol{x})=0$ is a material surface. It can be represented in the Lagrangian coordinates as $\varphi(t, \boldsymbol{x})=\varphi_{0}(\boldsymbol{X})$, which implies that $\delta \varphi=0$. 
Also,

$$
\delta\left(\frac{\partial \varphi}{\partial \boldsymbol{x}}\right)=\delta\left(\frac{\partial \varphi}{\partial \boldsymbol{X}} \boldsymbol{F}^{-1}\right)=\frac{\partial \delta \varphi}{\partial \boldsymbol{x}}-\frac{\partial \varphi}{\partial \boldsymbol{x}} \frac{\partial \zeta}{\partial \boldsymbol{x}}=-\frac{\partial \varphi}{\partial \boldsymbol{x}} \frac{\partial \zeta}{\partial \boldsymbol{x}} .
$$

Here we used the following expression for the variation of $\boldsymbol{F}^{-1}$ coming from the relation $\boldsymbol{F}^{-1} \boldsymbol{F}=\boldsymbol{I}$ :

$$
\delta \boldsymbol{F}^{-1}=-\boldsymbol{F}^{-1} \frac{\partial \zeta}{\partial \boldsymbol{x}}
$$

One also has

$$
\delta|\nabla \varphi|=\frac{(\nabla \varphi)^{T} \delta \nabla \varphi}{|\nabla \varphi|} .
$$

Finally, taking the variation of $\boldsymbol{n}=\nabla \varphi /|\nabla \varphi|$, one can obtain

$$
\delta \boldsymbol{n}=\left(\boldsymbol{n}^{T} \boldsymbol{n}-\boldsymbol{I}\right)\left(\frac{\partial \zeta}{\partial \boldsymbol{x}}\right)^{T} \boldsymbol{n}=-\boldsymbol{P}\left(\frac{\partial \zeta}{\partial \boldsymbol{x}}\right)^{T} \boldsymbol{n} .
$$

Proof of relation (13).

$$
\delta\left(\boldsymbol{F}^{-1} \boldsymbol{n}\right)=\delta\left(\boldsymbol{F}^{-1}\right) \boldsymbol{n}+\boldsymbol{F}^{-1} \delta \boldsymbol{n}=-\boldsymbol{F}^{-1} \frac{\partial \zeta}{\partial \boldsymbol{x}} \boldsymbol{n}+\boldsymbol{F}^{-1} \delta \boldsymbol{n} .
$$

Proof of relation (14).

$$
\delta\left(\frac{\partial \boldsymbol{n}}{\partial \boldsymbol{x}}\right)=\delta\left(\frac{\partial \boldsymbol{n}}{\partial \boldsymbol{X}} \boldsymbol{F}^{-1}\right)=\frac{\partial \delta \boldsymbol{n}}{\partial \boldsymbol{X}} \boldsymbol{F}^{-1}+\frac{\partial \boldsymbol{n}}{\partial \boldsymbol{X}} \delta \boldsymbol{F}^{-1}=\frac{\partial \delta \boldsymbol{n}}{\partial \boldsymbol{x}}-\frac{\partial \boldsymbol{n}}{\partial \boldsymbol{x}} \frac{\partial \zeta}{\partial \boldsymbol{x}} .
$$

We denote by $\sigma$ the energy per unit area of surface $S_{t}$. The variation of $\sigma$ is $\delta \sigma$. This variation depends on the physical problem through the dependence of $\sigma$ on geometrical and thermodynamical parameters. For now, we do not need to know this variation in explicit form; the variation will be given further. The next lemma gives the variation of the surface potential energy [Gouin 2014a; 2014b].

Lemma 3. Let us consider a material surface $S_{t}$ of boundary edge $C_{t}$. The variation of surface energy

$$
E=\iint_{S_{t}} \sigma d s
$$

is

$$
\delta E=\iint_{S_{t}}\left[\delta \sigma-\left(\nabla_{\mathrm{tg}}^{T} \sigma+\sigma H \boldsymbol{n}^{T}\right) \zeta\right] d s+\int_{C_{t}} \sigma \boldsymbol{n}^{\prime T} \zeta d l,
$$

where $d s, d l$ are the surface and line measures, respectively. ${ }^{1}$

${ }^{1}$ It is interesting to remark that the combination $\hat{\delta} \sigma=\delta \sigma-\left(\nabla_{\operatorname{tg}}^{T} \sigma\right) \zeta$ is the variation of $\sigma$ at fixed Eulerian coordinates. Indeed, since the symbol $\delta$ means the variation at fixed Lagrangian coordinates, and $\hat{\delta}$ is the variation at fixed Eulerian coordinates, this formula is a natural general relation between two types of variations [Gavrilyuk and Gouin 1999; Gavrilyuk 2011]. 
Proof. We suppose that the unit normal vector field is locally extended in the vicinity of $S_{t}$. For any vector field $\boldsymbol{w}$ one has

$$
\operatorname{rot}(\boldsymbol{n} \times \boldsymbol{w})=\boldsymbol{n} \operatorname{div} \boldsymbol{w}-\boldsymbol{w} \operatorname{div} \boldsymbol{n}+\frac{\partial \boldsymbol{n}}{\partial \boldsymbol{x}} \boldsymbol{w}-\frac{\partial \boldsymbol{w}}{\partial \boldsymbol{x}} \boldsymbol{n} .
$$

From relation $\boldsymbol{n}^{T} \boldsymbol{n}=1$, we obtain $\boldsymbol{n}^{T} \frac{\partial \boldsymbol{n}}{\partial \boldsymbol{x}}=0$. Using the definition of $H$ (that is, $H=-\operatorname{div} \boldsymbol{n})$, we deduce on $S_{t}$

$$
\boldsymbol{n}^{T} \operatorname{rot}(\boldsymbol{n} \times \boldsymbol{w})=\operatorname{div} \boldsymbol{w}+H \boldsymbol{n}^{T} \boldsymbol{w}-\boldsymbol{n}^{T} \frac{\partial \boldsymbol{w}}{\partial \boldsymbol{x}} \boldsymbol{n} .
$$

The surface energy is given by

$$
E=\iint_{S_{t}} \sigma\left|d_{1} x \wedge d_{2} x\right|,
$$

where $d_{i} \boldsymbol{x}=\frac{\partial \boldsymbol{x}}{\partial s_{i}} d s_{i}(i=1,2)$ and $s_{i}$ are curvilinear coordinates on $S_{t}$. This integral can also be written as

$$
E=\iint_{S_{t}} \sigma \operatorname{det}\left(\boldsymbol{n}, d_{1} \boldsymbol{x}, d_{2} \boldsymbol{x}\right)=\iint_{S_{0}} \sigma \operatorname{det}\left(\boldsymbol{F} \boldsymbol{F}^{-1} \boldsymbol{n}, \boldsymbol{F} d_{10} \boldsymbol{X}, \boldsymbol{F} d_{20} \boldsymbol{X}\right) .
$$

Here $d_{i 0} \boldsymbol{X}=\frac{\partial \boldsymbol{X}}{\partial s_{i 0}} d s_{i 0}$ and $s_{i 0}$ are the corresponding curvilinear coordinates on $S_{0}$. Finally,

$$
E=\iint_{S_{0}} \sigma(\operatorname{det} \boldsymbol{F}) \operatorname{det}\left(\boldsymbol{F}^{-1} \boldsymbol{n}, d_{10} \boldsymbol{X}, d_{20} \boldsymbol{X}\right)=\iint_{S_{0}} \sigma \operatorname{det}\left((\operatorname{det} \boldsymbol{F}) \boldsymbol{F}^{-1} \boldsymbol{n}, d_{10} \boldsymbol{X}, d_{20} \boldsymbol{X}\right) .
$$

Let us remark that $(\operatorname{det} \boldsymbol{F}) \boldsymbol{F}^{-1} \boldsymbol{n}$ is the image of $\boldsymbol{n}$ and is not the normal vector to $S_{0}$ because $\boldsymbol{F}$ is not an orthogonal transformation.

One has

$$
\delta E=\iint_{S_{0}} \delta \sigma \operatorname{det} \boldsymbol{F} \operatorname{det}\left(\boldsymbol{F}^{-1} \boldsymbol{n}, d_{10} \boldsymbol{X}, d_{20} \boldsymbol{X}\right)+\iint_{S_{0}} \sigma \delta\left(\operatorname{det} \boldsymbol{F} \operatorname{det}\left(\boldsymbol{F}^{-1} \boldsymbol{n}, d_{10} \boldsymbol{X}, d_{20} \boldsymbol{X}\right)\right) .
$$

Using Lemma 2, one gets

$$
\begin{aligned}
& \iint_{S_{0}} \sigma \delta\left(\operatorname{det} \boldsymbol{F} \operatorname{det}\left(\boldsymbol{F}^{-1} \boldsymbol{n}, d_{10} \boldsymbol{X}, d_{20} \boldsymbol{X}\right)\right) \\
& =\iint_{S_{t}} \sigma \operatorname{div} \zeta \operatorname{det}\left(\boldsymbol{n}, d_{1} \boldsymbol{x}, d_{2} \boldsymbol{x}\right)+\sigma \operatorname{det}\left(\delta \boldsymbol{n}, d_{1} \boldsymbol{x}, d_{2} \boldsymbol{x}\right)-\sigma \operatorname{det}\left(\frac{\partial \zeta}{\partial \boldsymbol{x}} \boldsymbol{n}, d_{1} \boldsymbol{x}, d_{2} \boldsymbol{x}\right) \\
& =\iint_{S_{t}}\left(\operatorname{div}(\sigma \zeta)-\left(\nabla^{T} \sigma\right) \zeta-\sigma \boldsymbol{n}^{T} \frac{\partial \zeta}{\partial \boldsymbol{x}} \boldsymbol{n}\right) d s .
\end{aligned}
$$

Relation (15) yields

$$
\operatorname{div}(\sigma \zeta)+\sigma H \boldsymbol{n}^{T} \zeta-\boldsymbol{n}^{T} \frac{\partial(\sigma \zeta)}{\partial \boldsymbol{x}} \boldsymbol{n}=\boldsymbol{n}^{T} \operatorname{rot}(\sigma \boldsymbol{n} \times \zeta)
$$


It implies

$$
\begin{aligned}
\iint_{S_{0}} \sigma \delta\left(\operatorname{det} \boldsymbol{F} \operatorname{det}\left(\boldsymbol{F}^{-1} \boldsymbol{n}, d_{10} \boldsymbol{X}, d_{20} \boldsymbol{X}\right)\right) \\
=\iint_{S_{t}}-\left(\sigma H \boldsymbol{n}^{T}+\left(\nabla^{T} \sigma\right) \boldsymbol{P}\right) \zeta d s+\iint_{S_{t}} \boldsymbol{n}^{T} \operatorname{rot}(\sigma \boldsymbol{n} \times \zeta) d s .
\end{aligned}
$$

Since $\boldsymbol{P} \nabla \sigma \equiv \nabla_{\mathrm{tg}} \sigma$, one has

$$
\iint_{S_{t}} \boldsymbol{n}^{T} \operatorname{rot}(\sigma \boldsymbol{n} \times \zeta) d s=\int_{C_{t}} \operatorname{det}(\boldsymbol{t}, \sigma \boldsymbol{n}, \zeta) d l=\int_{C_{t}} \sigma \boldsymbol{n}^{\prime T} \zeta d l
$$

and we obtain Lemma 3.

Lemma 4. Let $\sigma$ be a function of curvature tensor $\boldsymbol{R}$, or equivalently, a function of $H$ and $K$. Then,

$$
\frac{\partial \sigma}{\partial \boldsymbol{R}}=a \boldsymbol{I}+b \boldsymbol{R} \quad \text { with } a=\frac{\partial \sigma}{\partial H}+H \frac{\partial \sigma}{\partial K} \text { and } b=-\frac{\partial \sigma}{\partial K},
$$

where for the sake of simplicity, we indifferently write $\sigma(\boldsymbol{R})$ or $\sigma(H, K)$. In particular, this implies

$$
\boldsymbol{n}^{T} \frac{\partial \sigma}{\partial \boldsymbol{R}} \frac{\partial \boldsymbol{n}}{\partial \boldsymbol{x}}=\mathbf{0} .
$$

Proof. Since $H=\operatorname{tr} \boldsymbol{R}, 2 K=(\operatorname{tr} \boldsymbol{R})^{2}-\operatorname{tr}\left(\boldsymbol{R}^{2}\right)$, and

one gets

$$
\frac{\partial \operatorname{tr}\left(\boldsymbol{R}^{k}\right)}{\partial \boldsymbol{R}}=k \boldsymbol{R}^{k-1},
$$

$$
\frac{\partial \sigma}{\partial \boldsymbol{R}}=\left(\frac{\partial \sigma}{\partial H}+H \frac{\partial \sigma}{\partial K}\right) \boldsymbol{I}-\frac{\partial \sigma}{\partial K} \boldsymbol{R}
$$

Since

we obtain

$$
\boldsymbol{R}=-\frac{\partial \boldsymbol{n}}{\partial \boldsymbol{x}} \boldsymbol{P} \quad \text { and } \quad \frac{\partial \sigma}{\partial \boldsymbol{R}}=a \boldsymbol{I}+b \boldsymbol{R}
$$

$$
\boldsymbol{n}^{T} \frac{\partial \sigma}{\partial \boldsymbol{R}} \frac{\partial \boldsymbol{n}}{\partial \boldsymbol{x}}=a \boldsymbol{n}^{T} \frac{\partial \boldsymbol{n}}{\partial \boldsymbol{x}}-b \boldsymbol{n}^{T}\left(\frac{\partial \boldsymbol{n}}{\partial \boldsymbol{x}}\right)^{2}=\mathbf{0} .
$$

\section{Variation of $\sigma$}

This is a key part of the paper. The variation of the surface energy per unit area is obtained in the general case $\sigma=\sigma(H, K)$. The membrane is determined by a surface $S_{t}$ having a closed contact line $C_{t}$ on a rigid surface $\mathscr{S}=S_{1} \cup S_{2}$ (see Figure 1). The dependence on other parameters such as concentrations of surfactants on the membranes can further be taken into account as in [Gouin 2014a; Steigmann and Li 1995]. 


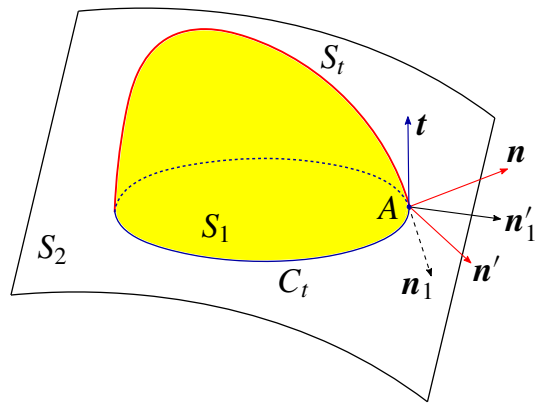

Figure 1. A drop lies on solid surface $\mathscr{S}=S_{1} \cup S_{2} ; S_{t}$ is a free surface; $\boldsymbol{n}_{1}$ and $\boldsymbol{n}$ are the external unit normal vectors to $S_{1}$ and $S_{t}$, respectively. Contact line $C_{t}$ separates $S_{1}$ and $S_{2}, t$ is the unit tangent vector to $C_{t}$ on $\mathscr{S}$. Vectors $\boldsymbol{n}_{1}^{\prime}=\boldsymbol{n}_{1} \times \boldsymbol{t}$ and $\boldsymbol{n}^{\prime}=\boldsymbol{t} \times \boldsymbol{n}$ are the binormals to $C_{t}$ relative to $\mathscr{S}$ and $S_{t}$ at point $A$ of $C_{t}$, respectively.

Lemma 5. The variation of surface energy $\sigma(\boldsymbol{R})$ is given by the relation

$$
\delta \sigma=-\operatorname{div}_{\operatorname{tg}}\left(\frac{\partial \sigma}{\partial \boldsymbol{R}} \boldsymbol{R} \boldsymbol{\zeta}+\boldsymbol{P} \frac{\partial \sigma}{\partial \boldsymbol{R}} \delta \boldsymbol{n}\right)+\operatorname{div}_{\operatorname{tg}}\left(\frac{\partial \sigma}{\partial \boldsymbol{R}} \boldsymbol{R}\right) \zeta+\operatorname{div}_{\operatorname{tg}}\left(\boldsymbol{P} \frac{\partial \sigma}{\partial \boldsymbol{R}}\right) \delta \boldsymbol{n} .
$$

Proof. Using Lemma 2, we have

$$
\delta \boldsymbol{R}=-\delta\left(\frac{\partial \boldsymbol{n}}{\partial \boldsymbol{x}} \boldsymbol{P}\right)=-\left(\frac{\partial \delta \boldsymbol{n}}{\partial \boldsymbol{x}}-\frac{\partial \boldsymbol{n}}{\partial \boldsymbol{x}} \frac{\partial \zeta}{\partial \boldsymbol{x}}\right) \boldsymbol{P}+\frac{\partial \boldsymbol{n}}{\partial \boldsymbol{x}} \delta\left(\boldsymbol{n} \boldsymbol{n}^{T}\right) .
$$

By taking account of (12) and $\delta\left(\boldsymbol{n} \boldsymbol{n}^{T}\right)=\delta \boldsymbol{n} \boldsymbol{n}^{T}+\boldsymbol{n} \delta \boldsymbol{n}^{T}$, we get

$$
\delta \boldsymbol{R}=-\frac{\partial \delta \boldsymbol{n}}{\partial \boldsymbol{x}} \boldsymbol{P}+\frac{\partial \boldsymbol{n}}{\partial \boldsymbol{x}} \frac{\partial \zeta}{\partial \boldsymbol{x}} \boldsymbol{P}-\frac{\partial \boldsymbol{n}}{\partial \boldsymbol{x}} \boldsymbol{P}\left(\frac{\partial \zeta}{\partial \boldsymbol{x}}\right)^{T} \boldsymbol{n} \boldsymbol{n}^{T}-\frac{\partial \boldsymbol{n}}{\partial \boldsymbol{x}} \boldsymbol{n} \boldsymbol{n}^{T} \frac{\partial \zeta}{\partial \boldsymbol{x}} \boldsymbol{P} .
$$

We deduce

$$
\begin{aligned}
\delta \sigma & =\operatorname{tr}\left(\frac{\partial \sigma}{\partial \boldsymbol{R}} \delta \boldsymbol{R}\right) \\
& =\operatorname{tr}\left[\frac{\partial \sigma}{\partial \boldsymbol{R}}\left(-\frac{\partial \delta \boldsymbol{n}}{\partial \boldsymbol{x}} \boldsymbol{P}+\frac{\partial \boldsymbol{n}}{\partial \boldsymbol{x}} \frac{\partial \zeta}{\partial \boldsymbol{x}} \boldsymbol{P}-\frac{\partial \boldsymbol{n}}{\partial \boldsymbol{x}} \boldsymbol{P}\left(\frac{\partial \zeta}{\partial \boldsymbol{x}}\right)^{T} \boldsymbol{n} \boldsymbol{n}^{T}-\frac{\partial \boldsymbol{n}}{\partial \boldsymbol{x}} \boldsymbol{n} \boldsymbol{n}^{T} \frac{\partial \zeta}{\partial \boldsymbol{x}} \boldsymbol{P}\right)\right] .
\end{aligned}
$$

From (17), we get $\boldsymbol{n} \boldsymbol{n}^{T} \frac{\partial \sigma}{\partial \boldsymbol{R}} \frac{\partial \boldsymbol{n}}{\partial x} \frac{\partial \zeta}{\partial \boldsymbol{x}}=0$ and $\boldsymbol{n} \boldsymbol{n}^{T} \frac{\partial \sigma}{\partial \boldsymbol{R}} \frac{\partial \boldsymbol{n}}{\partial \boldsymbol{x}} \boldsymbol{n} \boldsymbol{n}^{T} \frac{\partial \zeta}{\partial \boldsymbol{x}}=0$.

Consequently, $\frac{\partial \sigma}{\partial \boldsymbol{R}} \frac{\partial \boldsymbol{n}}{\partial \boldsymbol{x}} \boldsymbol{P} \frac{\partial \boldsymbol{\zeta}}{\partial \boldsymbol{x}}=-\frac{\partial \sigma}{\partial \boldsymbol{R}} \boldsymbol{R} \frac{\partial \zeta}{\partial \boldsymbol{x}}$, which implies

$$
\begin{aligned}
\delta \sigma & =-\operatorname{tr}\left[\boldsymbol{P} \frac{\partial \sigma}{\partial \boldsymbol{R}} \frac{\partial \delta \boldsymbol{n}}{\partial \boldsymbol{x}}+\frac{\partial \sigma}{\partial \boldsymbol{R}} \boldsymbol{R} \frac{\partial \zeta}{\partial \boldsymbol{x}}\right] \\
& =-\operatorname{div}\left(\boldsymbol{P} \frac{\partial \sigma}{\partial \boldsymbol{R}} \delta \boldsymbol{n}\right)+\operatorname{div}\left(\boldsymbol{P} \frac{\partial \sigma}{\partial \boldsymbol{R}}\right) \delta \boldsymbol{n}-\operatorname{div}\left(\frac{\partial \sigma}{\partial \boldsymbol{R}} \boldsymbol{R} \zeta\right)+\operatorname{div}\left(\frac{\partial \sigma}{\partial \boldsymbol{R}} \boldsymbol{R}\right) \zeta
\end{aligned}
$$


By taking account of (5), we get

$\delta \sigma=-\operatorname{div}_{\operatorname{tg}}\left(\boldsymbol{P} \frac{\partial \sigma}{\partial \boldsymbol{R}} \delta \boldsymbol{n}\right)+\operatorname{div}_{\mathrm{tg}}\left(\boldsymbol{P} \frac{\partial \sigma}{\partial \boldsymbol{R}}\right) \delta \boldsymbol{n}-\operatorname{div}_{\mathrm{tg}}\left(\frac{\partial \sigma}{\partial \boldsymbol{R}} \boldsymbol{R} \boldsymbol{\zeta}\right)+\operatorname{div}_{\mathrm{tg}}\left(\frac{\partial \sigma}{\partial \boldsymbol{R}} \boldsymbol{R}\right) \boldsymbol{\zeta}$,

and relation (19) is proven.

Now, we have to study term $\operatorname{div}_{\operatorname{tg}}\left(\boldsymbol{P} \frac{\partial \sigma}{\partial \boldsymbol{R}}\right) \delta \boldsymbol{n}$.

Lemma 6. $\operatorname{div}_{\operatorname{tg}}\left(\boldsymbol{P} \frac{\partial \sigma}{\partial \boldsymbol{R}}\right) \delta \boldsymbol{n}=-\operatorname{div}_{\operatorname{tg}}\left[\boldsymbol{P} \operatorname{div}_{\operatorname{tg}}^{T}\left(\boldsymbol{P} \frac{\partial \sigma}{\partial \boldsymbol{R}}\right) \boldsymbol{n}^{T} \zeta\right]$

$$
+\operatorname{div}_{\operatorname{tg}}\left[\boldsymbol{P} \operatorname{div}_{\operatorname{tg}}^{T}\left(\boldsymbol{P} \frac{\partial \sigma}{\partial \boldsymbol{R}}\right)\right] \boldsymbol{n}^{T} \zeta-\operatorname{div}_{\operatorname{tg}}\left(\boldsymbol{P} \frac{\partial \sigma}{\partial \boldsymbol{R}}\right) \boldsymbol{R} \zeta .
$$

Proof. Using relation (12), one obtains

$$
\begin{aligned}
\operatorname{div}_{\operatorname{tg}}\left(\boldsymbol{P} \frac{\partial \sigma}{\partial \boldsymbol{R}}\right) \delta \boldsymbol{n} & =-\operatorname{div}_{\operatorname{tg}}\left(\boldsymbol{P} \frac{\partial \sigma}{\partial \boldsymbol{R}}\right) \boldsymbol{P}\left(\frac{\partial \zeta}{\partial \boldsymbol{x}}\right)^{T} \boldsymbol{n} \\
& =-\operatorname{div}_{\operatorname{tg}}\left(\boldsymbol{P} \frac{\partial \sigma}{\partial \boldsymbol{R}}\right) \boldsymbol{P}\left[\left(\frac{\partial\left(\boldsymbol{n}^{T} \zeta\right)}{\partial \boldsymbol{x}}\right)^{T}-\left(\frac{\partial \boldsymbol{n}}{\partial \boldsymbol{x}}\right)^{T} \zeta\right] \\
& =-\operatorname{div}_{\operatorname{tg}}\left(\boldsymbol{P} \frac{\partial \sigma}{\partial \boldsymbol{R}}\right) \nabla_{\mathrm{tg}}\left(\boldsymbol{n}^{T} \zeta\right)-\operatorname{div}_{\mathrm{tg}}\left(\boldsymbol{P} \frac{\partial \sigma}{\partial \boldsymbol{R}}\right) \boldsymbol{R} \boldsymbol{\zeta} \\
& =\operatorname{div}_{\mathrm{tg}}\left[\boldsymbol{P} \operatorname{div}_{\operatorname{tg}}^{T}\left(\boldsymbol{P} \frac{\partial \sigma}{\partial \boldsymbol{R}}\right)\right] \boldsymbol{n}^{T} \boldsymbol{\zeta}-\operatorname{div}_{\operatorname{tg}}\left(\boldsymbol{P} \frac{\partial \sigma}{\partial \boldsymbol{R}}\right) \boldsymbol{R} \boldsymbol{C} \\
& -\operatorname{div}_{\mathrm{tg}}\left[\boldsymbol{P} \operatorname{div}_{\mathrm{tg}}^{T}\left(\boldsymbol{P} \frac{\partial \sigma}{\partial \boldsymbol{R}}\right) \boldsymbol{n}^{T} \zeta\right] .
\end{aligned}
$$

Now, from Lemma 3 and (19), we obtain the following fundamental lemma.

Lemma 7. The variation of surface energy $E=\iint_{S_{t}} \sigma d s$, where $S_{t}$ has an oriented boundary line $C_{t}$ with tangent unit vector $\boldsymbol{t}$ and binormal unit vector $\boldsymbol{n}^{\prime}=\boldsymbol{t} \times \boldsymbol{n}$, is given by the relation

$$
\begin{aligned}
& \delta E=\iint_{S_{t}}\left[\operatorname{div}_{\operatorname{tg}}\left(\frac{\partial \sigma}{\partial \boldsymbol{R}} \boldsymbol{R}\right)-\operatorname{div}_{\operatorname{tg}}\left(\boldsymbol{P} \frac{\partial \sigma}{\partial \boldsymbol{R}}\right) \boldsymbol{R}+\operatorname{div}_{\operatorname{tg}}\left(\boldsymbol{P} \operatorname{div}_{\operatorname{tg}}^{T}\left(\boldsymbol{P} \frac{\partial \sigma}{\partial \boldsymbol{R}}\right)\right) \boldsymbol{n}^{T}\right. \\
&\left.-\sigma H \boldsymbol{n}^{T}-\nabla_{\mathrm{tg}}^{T} \sigma\right] \zeta d s \\
&+\int_{C_{t}} \boldsymbol{n}^{\prime T}\left\{\left[\sigma \boldsymbol{I}-\frac{\partial \sigma}{\partial \boldsymbol{R}} \boldsymbol{R}-\operatorname{div}_{\mathrm{tg}}^{T}\left(\boldsymbol{P} \frac{\partial \sigma}{\partial \boldsymbol{R}}\right) \boldsymbol{n}^{T}\right] \zeta+\frac{\partial \sigma}{\partial \boldsymbol{R}} \boldsymbol{P}\left(\frac{\partial \zeta}{\partial \boldsymbol{x}}\right)^{T} \boldsymbol{n}\right\} d l .
\end{aligned}
$$

Proof. By taking account of Lemmas 5 and 6, we get

$$
\begin{aligned}
\delta \sigma=-\operatorname{div}_{\mathrm{tg}} & {\left[\frac{\partial \sigma}{\partial \boldsymbol{R}} \boldsymbol{R} \zeta+\boldsymbol{P} \frac{\partial \sigma}{\partial \boldsymbol{R}} \delta \boldsymbol{n}+\boldsymbol{P} \operatorname{div}_{\operatorname{tg}}^{T}\left(\boldsymbol{P} \frac{\partial \sigma}{\partial \boldsymbol{R}}\right) \boldsymbol{n}^{T} \boldsymbol{\zeta}\right] } \\
+ & {\left[\operatorname{div}_{\mathrm{tg}}\left(\frac{\partial \sigma}{\partial \boldsymbol{R}} \boldsymbol{R}\right)-\operatorname{div}_{\mathrm{tg}}\left(\boldsymbol{P} \frac{\partial \sigma}{\partial \boldsymbol{R}}\right) \boldsymbol{R}+\operatorname{div}_{\mathrm{tg}}\left(\boldsymbol{P} \operatorname{div}_{\mathrm{tg}}^{T}\left(\boldsymbol{P} \frac{\partial \sigma}{\partial \boldsymbol{R}}\right)\right) \boldsymbol{n}^{T}\right] \boldsymbol{\zeta} . }
\end{aligned}
$$


By using (6) and Lemma 3 associated with the Stokes formula, and property $\boldsymbol{n}^{\prime T} \boldsymbol{P}=\boldsymbol{n}^{\prime T}$, we obtain

$$
\begin{array}{r}
\delta E=\iint_{S_{t}}\left[\operatorname{div}_{\operatorname{tg}}\left(\frac{\partial \sigma}{\partial \boldsymbol{R}} \boldsymbol{R}\right)-\operatorname{div}_{\operatorname{tg}}\left(\boldsymbol{P} \frac{\partial \sigma}{\partial \boldsymbol{R}}\right) \boldsymbol{R}+\operatorname{div}_{\operatorname{tg}}\left(\boldsymbol{P} \operatorname{div}_{\operatorname{tg}}^{T}\left(\boldsymbol{P} \frac{\partial \sigma}{\partial \boldsymbol{R}}\right)\right) \boldsymbol{n}^{T}\right. \\
\left.-\sigma H \boldsymbol{n}^{T}-\nabla_{\mathrm{tg}}^{T} \sigma\right] \zeta d s \\
+\int_{C_{t}} \boldsymbol{n}^{\prime T}\left\{\left[\sigma \boldsymbol{I}-\frac{\partial \sigma}{\partial \boldsymbol{R}} \boldsymbol{R}-\operatorname{div}_{\operatorname{tg}}^{T}\left(\boldsymbol{P} \frac{\partial \sigma}{\partial \boldsymbol{R}}\right) \boldsymbol{n}^{T}\right] \zeta-\frac{\partial \sigma}{\partial \boldsymbol{R}} \delta \boldsymbol{n}\right\} d l .
\end{array}
$$

From Lemma 2 we deduce

$$
-\boldsymbol{n}^{\prime T} \frac{\partial \sigma}{\partial \boldsymbol{R}} \delta \boldsymbol{n}=\boldsymbol{n}^{\prime T} \frac{\partial \sigma}{\partial \boldsymbol{R}} \boldsymbol{P}\left(\frac{\partial \zeta}{\partial \boldsymbol{x}}\right)^{T} \boldsymbol{n},
$$

which proves Lemma 7.

\section{Equations of motion and shape equation}

The vesicle occupies domain $D_{t}$ with a free boundary $S_{t}$ which is the membrane surface, and $S_{1}$ which belongs to the rigid surface $\mathscr{Y}=S_{1} \cup S_{2} . S_{1}$ denotes the footprint of $D_{t}$ on $\mathscr{Y}$, and $C_{t}$ is the closed edge (contact line) between $S_{1}$ and $S_{2}$ (see Figure 1).

We denote by $\boldsymbol{n}_{1}$ the external unit normal to $S_{1}$ along contact line $C_{t}$. Then denoting $t_{1}=-t$, one has

$$
\boldsymbol{n}_{1}^{\prime}=\boldsymbol{t}_{1} \times \boldsymbol{n}_{1}=\boldsymbol{n}_{1} \times \boldsymbol{t} .
$$

The surface energy of membrane $S_{t}$ is denoted $\sigma$. Solid surfaces $S_{1}$ and $S_{2}$ have constant surface energies denoted $\sigma_{1}$ and $\sigma_{2}$. The geometrical notations are shown in Figure 1.

One can formulate the virtual work principle in the form [Germain 1973; Gouin 2007]

$$
\delta \mathscr{A}_{e}+\delta \mathscr{A}_{i}-\delta \mathscr{E}=0,
$$

where $\delta \mathscr{A}_{e}$ is the virtual work of external forces, $\delta \mathscr{A}_{i}$ is the virtual work of inertial forces, and $\delta \mathscr{E}$ is the variation of the total energy. The energy $\mathscr{E}$ is taken in the form

$$
\mathscr{E}=\iiint_{D_{t}} \rho \varepsilon d v+\iint_{S_{t}} \sigma d s+\iint_{S_{1}} \sigma_{1} d s,
$$

where specific internal energy $\varepsilon$ is a function of density $\rho$. As we mentioned before, one can also include in this dependence several scalar quantities which are transported by the flow (specific entropy, mass fractions of surfactants, etc.). From 
Lemma 2, (11), and the mass conservation law

$$
\rho \operatorname{det} \boldsymbol{F}=\rho_{0}(\boldsymbol{X}),
$$

we obtain the variation of the specific energy and density at fixed Lagrangian coordinates in the form

$$
\delta \varepsilon=\frac{p}{\rho^{2}} \delta \rho \quad \text { with } \delta \rho=-\rho \operatorname{div} \zeta,
$$

where $p$ is the thermodynamical pressure. Consequently, the variation of the first term is [Berdichevsky 2009; Gavrilyuk 2011; Serrin 1959]

$$
\begin{aligned}
\delta \iiint_{D_{t}} \rho \varepsilon d v & =\delta \iiint_{D_{0}} \rho_{0} \varepsilon d v_{0}=\iiint_{D_{0}} \rho_{0} \delta \varepsilon d v_{0} \\
& =\iiint_{D_{t}} \rho \delta \varepsilon d v=-\iiint_{D_{t}} p \operatorname{div} \zeta d v .
\end{aligned}
$$

The variation of the surface energy is given in Lemma 3. The third term is the surface energy of $S_{1}$ with energy $\sigma_{1}$ per unit surface. The virtual work of the external forces is given in the form

$$
\delta \mathscr{A}_{e}=\iiint_{D_{t}} \rho \boldsymbol{f}^{T} \zeta d v+\iint_{S_{t}} \boldsymbol{T}^{T} \zeta d s+\int_{C_{t}} \sigma_{2} \boldsymbol{n}_{1}^{\prime T} \zeta d s,
$$

where $\rho \boldsymbol{f}$ is the volume external force in $D_{t}, \boldsymbol{T}$ is the external stress vector at the free surface $S_{t}$, and $\sigma_{2} \boldsymbol{n}_{1}^{\prime}$ is the line tension vector exerted on $C_{t}$. The last term on the right-hand side comes from Lemma 3 which can be also applied for rigid surfaces. Finally,

$$
\delta \mathscr{A}_{i}=-\iiint_{D_{t}} \rho \boldsymbol{a}^{T} \zeta d v
$$

is the virtual work of inertial force, where $\boldsymbol{a}$ is the acceleration. The virtual work of forces $\delta \mathscr{T}$ applied to the material volume $D_{t}$ is defined as

$$
\begin{gathered}
\delta \mathscr{T}=\iiint_{D_{t}}\left(-\rho \boldsymbol{a}^{T}+\rho \boldsymbol{f}^{T}-\nabla^{T} p\right) \zeta d v+\iint_{S_{1}}\left(p+H_{1} \sigma_{1}\right) \boldsymbol{n}_{1}^{T} \zeta d s \\
+\iint_{S_{t}}\left[-\operatorname{div}_{\operatorname{tg}}\left(\frac{\partial \sigma}{\partial \boldsymbol{R}} \boldsymbol{R}\right)+\operatorname{div}_{\operatorname{tg}}\left(\boldsymbol{P} \frac{\partial \sigma}{\partial \boldsymbol{R}}\right) \boldsymbol{R}\right. \\
\left.\quad-\operatorname{div}_{\operatorname{tg}}\left(\boldsymbol{P} \operatorname{div}_{\operatorname{tg}}^{T}\left(\boldsymbol{P} \frac{\partial \sigma}{\partial \boldsymbol{R}}\right)\right) \boldsymbol{n}^{T}+(p+H \sigma) \boldsymbol{n}^{T}+\nabla_{\mathrm{tg}}^{T} \sigma+\boldsymbol{T}^{T}\right] \zeta d s \\
-\int_{C_{t}}\left\{\left[\left(\sigma_{1}-\sigma_{2}\right) \boldsymbol{n}_{1}^{\prime T}+\sigma \boldsymbol{n}^{\prime T}-\boldsymbol{n}^{\prime T} \operatorname{div}_{\operatorname{tg}}^{T}\left(\boldsymbol{P} \frac{\partial \sigma}{\partial \boldsymbol{R}}\right) \boldsymbol{n}^{T}-\boldsymbol{n}^{\prime T} \frac{\partial \sigma}{\partial \boldsymbol{R}} \boldsymbol{R}\right] \zeta\right. \\
\left.+\boldsymbol{n}^{\prime T} \frac{\partial \sigma}{\partial \boldsymbol{R}} \boldsymbol{P}\left(\frac{\partial \zeta}{\partial \boldsymbol{x}}\right)^{T} \boldsymbol{n}\right\} d l .
\end{gathered}
$$


As usual, $H_{1}$ and $H$ are the sum of principle curvatures of surfaces $S_{1}$ and $S_{t}$, respectively. Terms on $D_{t}, S_{1}, S_{t}$ are in separable form with respect to the field $\zeta$. Expression (20) implies the equation of motion in $D_{t}$ and boundary conditions on surfaces $S_{1}, S_{t}$ [Schwartz 1966, Chapitre 3]. Virtual displacement $\zeta$ must be compatible with conditions of the problem; for example, $S_{1}$ is an external surface to domain $D_{t}$ and consequently $\zeta$ must be tangent to $S_{1}$. This notion is developed in [Berdichevsky 2009]. They are presented below.

6.1. Equation of motion. We consider virtual displacements $\zeta$ which vanish on the boundary of $D_{t}$. The fundamental lemma of virtual displacements yields

$$
\rho \boldsymbol{a}+\nabla p=\rho \boldsymbol{f},
$$

which is the classical Newton law in continuum mechanics.

6.2. Condition on surface $S_{1}$. Due to the fact that the surface $S_{1}$ is - a priorigiven, the virtual displacements must be compatible with the geometry of $S_{1}$. This means that the nonpenetration condition (slip condition) is verified:

$$
\boldsymbol{n}_{1}^{T} \zeta=0 .
$$

Constraint (22) is equivalent to the introduction of a Lagrange multiplier $\mathscr{P}_{1}$ into (20) where $\zeta$ is now a virtual displacement without constraint. The corresponding term on $S_{1}$ will be modified into

$$
\iint_{S_{1}}\left(p+H_{1} \sigma_{1}-\mathscr{P}_{1}\right) \boldsymbol{n}_{1}^{T} \zeta d s .
$$

Since the variation of $\zeta$ on $S_{1}$ is independent, (20) implies

$$
\mathscr{P}_{1}=p+H_{1} \sigma_{1} .
$$

This is the classical Laplace condition allowing us to obtain the normal stress component $\mathscr{P}_{1} \boldsymbol{n}_{1}$ exerted by surface $S_{1}$.

6.3. Extended shape equation. Taking account of (21) and (23), for all displacement $\zeta$ on moving membrane $S_{t}$, one has from (20)

$$
\begin{aligned}
\iint_{S_{t}}\left[-\operatorname{div}_{\mathrm{tg}}\right. & \left(\frac{\partial \sigma}{\partial \boldsymbol{R}} \boldsymbol{R}\right)+\operatorname{div}_{\mathrm{tg}}\left(\boldsymbol{P} \frac{\partial \sigma}{\partial \boldsymbol{R}}\right) \boldsymbol{R} \\
& \left.-\operatorname{div}_{\mathrm{tg}}\left(\boldsymbol{P} \operatorname{div}_{\mathrm{tg}}^{T}\left(\boldsymbol{P} \frac{\partial \sigma}{\partial \boldsymbol{R}}\right)\right) \boldsymbol{n}^{T}+(p+H \sigma) \boldsymbol{n}^{T}+\nabla_{\mathrm{tg}}^{T} \sigma+\boldsymbol{T}^{T}\right] \zeta d s=0 .
\end{aligned}
$$


It implies

$$
\begin{aligned}
\left\{p+H \sigma-\operatorname{div}_{\mathrm{tg}}\left[\boldsymbol{P} \operatorname{div}_{\mathrm{tg}}^{T}\left(\boldsymbol{P} \frac{\partial \sigma}{\partial \boldsymbol{R}}\right)\right]\right\} \boldsymbol{n} \\
+\nabla_{\mathrm{tg}} \sigma-\operatorname{div}_{\operatorname{tg}}^{T}\left(\frac{\partial \sigma}{\partial \boldsymbol{R}} \boldsymbol{R}\right)+\boldsymbol{R} \operatorname{div}_{\operatorname{tg}}^{T}\left(\boldsymbol{P} \frac{\partial \sigma}{\partial \boldsymbol{R}}\right)+\boldsymbol{T}=0 .
\end{aligned}
$$

Equation (24) is the most general form of the dynamical boundary condition on $S_{t}$. Due to the fact that surface energy $\sigma$ must be an isotropic function of curvature tensor $\boldsymbol{R}$, i.e., a function of two invariants $H$ and $K$, we obtain (for proof, see the Appendix) that the vector

$$
\nabla_{\mathrm{tg}} \sigma-\operatorname{div}_{\mathrm{tg}}^{T}\left(\frac{\partial \sigma}{\partial \boldsymbol{R}} \boldsymbol{R}\right)+\boldsymbol{R} \operatorname{div}_{\mathrm{tg}}^{T}\left(\boldsymbol{P} \frac{\partial \sigma}{\partial \boldsymbol{R}}\right)
$$

is normal to $S_{t}$ and consequently $\boldsymbol{T}$ can be written in the form

$$
\boldsymbol{T}=-\mathscr{P} \boldsymbol{n} .
$$

Here scalar $\mathscr{P}$ has the dimension of pressure.

One obtains from (44) (see the Appendix)

$$
\begin{aligned}
H \sigma-\Delta_{\mathrm{tg}} a-b \Delta_{\mathrm{tg}} H-\nabla_{\mathrm{tg}}^{T} b \nabla_{\mathrm{tg}} H & -\operatorname{div}_{\mathrm{tg}}\left(\boldsymbol{R} \nabla_{\mathrm{tg}} b\right) \\
& +\left(2 K-H^{2}\right) \frac{\partial \sigma}{\partial H}-H K \frac{\partial \sigma}{\partial K}=\mathscr{P}-p .
\end{aligned}
$$

Relation (25) is the normal component of (24).

It is important to underline that (24) is only expressed in the normal direction to $S_{t}$. This is not the case when surface energy $\sigma$ also depends on physicochemical characteristics of $S_{t}$, such as temperature or surfactants. In this last case, Marangoni effects can appear producing additive tangential terms to $S_{t}$.

Using Lemma 1 (second equation) and expressions of scalars $a$ and $b$ given by (16), we get the extended shape equation:

$$
\begin{aligned}
H\left(\sigma-K \frac{\partial \sigma}{\partial K}\right)+\left(2 K-H^{2}\right) & \frac{\partial \sigma}{\partial H}-\Delta_{\operatorname{tg}} \frac{\partial \sigma}{\partial H}-H \Delta_{\operatorname{tg}} \frac{\partial \sigma}{\partial K} \\
& -\nabla_{\operatorname{tg}}^{T} H \nabla_{\operatorname{tg}} \frac{\partial \sigma}{\partial K}+\operatorname{div}_{\operatorname{tg}}\left(\boldsymbol{R} \nabla_{\operatorname{tg}} \frac{\partial \sigma}{\partial K}\right)=\mathscr{P}-p .
\end{aligned}
$$

Equation (26) was also derived in [Rosso and Virga 1999] under the hypothesis (10) and the assumption of inextensibility of the membrane. Our derivation does not use these hypotheses. For example, the inextensibility property is not natural even in the case of incompressible fluids (at fixed volume, the surface of a three-dimensional body may vary). 


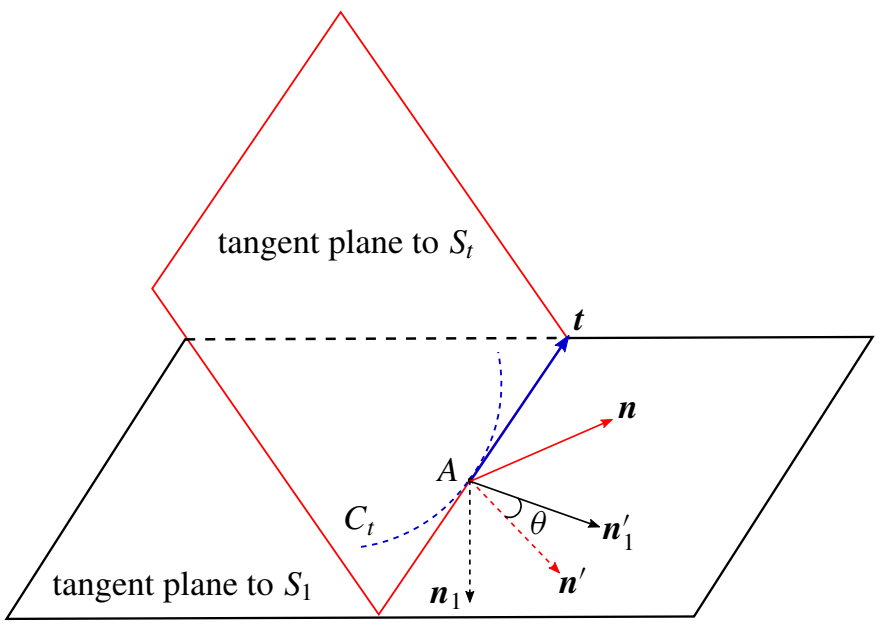

Figure 2. Tangent planes to membrane $S_{t}$ and solid surface $S_{1}$ : $\boldsymbol{n}_{1}$ and $\boldsymbol{n}$ are the unit normal vectors to $\mathscr{S}$ and $S_{t}$, external to the domain of the vesicle; contact line $C_{t}$ is shared between $\mathscr{S}$ and $S_{t}$ and $\boldsymbol{t}$ is the unit tangent vector to $C_{t}$ relative to $\boldsymbol{n} ; \boldsymbol{n}_{1}^{\prime}=\boldsymbol{n}_{1} \times \boldsymbol{t}$ and $\boldsymbol{n}^{\prime}=\boldsymbol{t} \times \boldsymbol{n}$ are binormals to $C_{t}$ relative to $\mathscr{S}$ and $S_{t}$ at point $A$, respectively. Angle $\theta=\left\langle\boldsymbol{n}^{\prime}, \boldsymbol{n}_{1}^{\prime}\right\rangle$. The normal plane to $C_{t}$ at $A$ contains vectors $\boldsymbol{n}, \boldsymbol{n}^{\prime}, \boldsymbol{n}_{1}, \boldsymbol{n}_{1}^{\prime}$.

6.4. Helfrich's shape equation. The Helfrich energy is given by (1). The shape equation (26) can immediately be written in the form

$$
\sigma_{0} H+\frac{\kappa}{2}\left(H-H_{0}\right)\left[4 K-H\left(H+H_{0}\right)\right]-\kappa \Delta_{\mathrm{tg}} H=\mathscr{P}-p,
$$

which is the classical form obtained by Helfrich. ${ }^{2}$

\section{Extended Young-Dupré condition on contact line $C_{t}$}

Let us denote by $\theta=\left\langle\boldsymbol{n}^{\prime}, \boldsymbol{n}_{1}^{\prime}\right\rangle=\pi+\left\langle\boldsymbol{n}, \boldsymbol{n}_{1}\right\rangle(\bmod 2 \pi)$ the Young angle between $S_{1}$ and $S_{t}$ (see Figure 2).

Due to the fact that $C_{t}$ belongs to $S_{1}$, the virtual displacement on $C_{t}$ is in the form

$$
\zeta=\alpha \boldsymbol{t}+\beta \boldsymbol{n}_{1}^{\prime},
$$

${ }^{2}$ Let us note that Helfrich considered the vesicle as an incompressible fluid. He also assumed that the membrane has a total constant area. Then, the virtual work can be expressed as

$$
\delta \mathcal{T}=\iiint_{D} \rho \boldsymbol{f}^{T} \zeta d v+\iint_{S} \boldsymbol{T}^{T} \zeta d s-\delta \iint_{S} \sigma d s+\lambda_{0} \delta \iint_{S} d s+\delta \iiint_{D} p \operatorname{div} \zeta d v,
$$

where the scalar $\lambda_{0}$ is a constant Lagrange multiplier and $p$ is a distributed Lagrange multiplier. The "shape equation" is similar to (27). 
where $\alpha$ and $\beta$ are two scalar fields defined on $S_{1}$. Let us remark that condition (28) expresses the nonpenetration condition (22) on $S_{1}$. Moreover, since $\boldsymbol{n}, \boldsymbol{n}_{1}, \boldsymbol{n}_{1}^{\prime}$ belong to the normal plane to $C_{t}$ at $A$ (see Figure 2), one has

$$
\boldsymbol{n}=\boldsymbol{n}_{1}^{\prime} \sin \theta-\boldsymbol{n}_{1} \cos \theta .
$$

But relation $\boldsymbol{\zeta}^{T} \boldsymbol{n}_{1}=0$ implies

$$
\boldsymbol{P}\left(\frac{\partial \zeta}{\partial \boldsymbol{x}}\right)^{T} \boldsymbol{n}_{1}+\boldsymbol{P}\left(\frac{\partial \boldsymbol{n}_{1}}{\partial \boldsymbol{x}}\right)^{T} \zeta=0
$$

Replacing (29) into (20) one has

$$
\begin{aligned}
& \delta \mathscr{T}=-\int_{C_{t}}\left\{\left[\left(\sigma_{1}-\sigma_{2}\right) \boldsymbol{n}_{1}^{\prime T}+\sigma \boldsymbol{n}^{\prime T}-\boldsymbol{n}^{\prime T} \operatorname{div}_{\operatorname{tg}}^{T}\left(\boldsymbol{P} \frac{\partial \sigma}{\partial \boldsymbol{R}}\right) \boldsymbol{n}^{T}-\boldsymbol{n}^{\prime T} \frac{\partial \sigma}{\partial \boldsymbol{R}} \boldsymbol{R}\right] \zeta\right. \\
&\left.+\boldsymbol{n}^{\prime T} \frac{\partial \sigma}{\partial \boldsymbol{R}} \boldsymbol{P}\left(\frac{\partial \zeta}{\partial \boldsymbol{x}}\right)^{T} \boldsymbol{n}\right\} d l \\
&=-\int_{C_{t}}\left\{\left[\left(\sigma_{1}-\sigma_{2}\right) \boldsymbol{n}_{1}^{\prime T}+\sigma \boldsymbol{n}^{\prime T}-\boldsymbol{n}^{\prime T} \operatorname{div}_{\operatorname{tg}}^{T}\left(\boldsymbol{P} \frac{\partial \sigma}{\partial \boldsymbol{R}}\right) \boldsymbol{n}^{T}-\boldsymbol{n}^{\prime T} \frac{\partial \sigma}{\partial \boldsymbol{R}} \boldsymbol{R}\right.\right. \\
&\left.\quad+\cos \theta \boldsymbol{n}^{\prime T} \frac{\partial \sigma}{\partial \boldsymbol{R}} \boldsymbol{P}\left(\frac{\partial \boldsymbol{n}_{1}}{\partial \boldsymbol{x}}\right)^{T}\right] \zeta \\
&\left.+\sin \theta \boldsymbol{n}^{\prime T} \frac{\partial \sigma}{\partial \boldsymbol{R}} \boldsymbol{P}\left(\frac{\partial \zeta}{\partial \boldsymbol{x}}\right)^{T} \boldsymbol{n}_{1}^{\prime}\right\} d l=0 .
\end{aligned}
$$

We choose now the virtual displacement in the form $\zeta=\beta \boldsymbol{n}_{1}^{\prime}$. One has

$$
\frac{\partial \zeta}{\partial \boldsymbol{x}}=\boldsymbol{n}_{1}^{\prime}(\nabla \beta)^{T}+\beta \frac{\partial \boldsymbol{n}_{1}^{\prime}}{\partial \boldsymbol{x}}, \quad\left(\frac{\partial \zeta}{\partial \boldsymbol{x}}\right)^{T}=\nabla \beta \boldsymbol{n}_{1}^{\prime T}+\beta\left(\frac{\partial \boldsymbol{n}_{1}^{\prime}}{\partial \boldsymbol{x}}\right)^{T} .
$$

Since $\left(\frac{\partial \boldsymbol{n}_{1}^{\prime}}{\partial \boldsymbol{x}}\right)^{T} \boldsymbol{n}_{1}^{\prime}=0$, it implies

$$
\left(\frac{\partial \zeta}{\partial \boldsymbol{x}}\right)^{T} \boldsymbol{n}_{1}^{\prime}=\nabla \beta
$$

The integral (30) becomes

$$
\begin{aligned}
\int_{C_{t}}\left\{\left[\left(\sigma_{1}-\sigma_{2}\right) \boldsymbol{n}_{1}^{\prime T}+\sigma \boldsymbol{n}^{\prime T}-\boldsymbol{n}^{\prime T} \operatorname{div}_{\operatorname{tg}}^{T}\left(\boldsymbol{P} \frac{\partial \sigma}{\partial \boldsymbol{R}}\right) \boldsymbol{n}^{T}-\boldsymbol{n}^{\prime T} \frac{\partial \sigma}{\partial \boldsymbol{R}} \boldsymbol{R}\right.\right. \\
\left.\left.+\cos \theta \boldsymbol{n}^{\prime T} \frac{\partial \sigma}{\partial \boldsymbol{R}} \boldsymbol{P}\left(\frac{\partial \boldsymbol{n}_{1}}{\partial \boldsymbol{x}}\right)^{T}\right] \boldsymbol{n}_{1}^{\prime} \beta+\sin \theta \boldsymbol{n}^{\prime T} \frac{\partial \sigma}{\partial \boldsymbol{R}} \boldsymbol{P} \nabla \beta\right\} d l=0 .
\end{aligned}
$$


Since $\beta$ and the components of $\nabla \beta$ can be chosen as independent, relation (31) implies two boundary conditions. The first condition on line $C_{t}$ is

$$
\sin \theta \boldsymbol{n}^{\prime T} \frac{\partial \sigma}{\partial \boldsymbol{R}} \boldsymbol{P}=0
$$

The second condition is

$$
\begin{aligned}
{\left[\left(\sigma_{1}-\sigma_{2}\right) \boldsymbol{n}_{1}^{\prime T}+\sigma \boldsymbol{n}^{\prime T}-\boldsymbol{n}^{\prime T}\right.} & \operatorname{div}_{\operatorname{tg}}^{T}\left(\boldsymbol{P} \frac{\partial \sigma}{\partial \boldsymbol{R}}\right) \boldsymbol{n}^{T} \\
& \left.-\boldsymbol{n}^{\prime T} \frac{\partial \sigma}{\partial \boldsymbol{R}} \boldsymbol{R}+\cos \theta \boldsymbol{n}^{\prime T} \frac{\partial \sigma}{\partial \boldsymbol{R}} \boldsymbol{P}\left(\frac{\partial \boldsymbol{n}_{1}}{\partial \boldsymbol{x}}\right)^{T}\right] \boldsymbol{n}_{1}^{\prime}=0 .
\end{aligned}
$$

The case $\sin \theta=0$ all along $C_{t}$ is degenerate. If $\theta=0$, this corresponds to a hydrophobic surface (the contact line is absent). If $\theta=\pi$, this corresponds to a complete wetting. In the last case $\boldsymbol{n}_{1}^{\prime}=-\boldsymbol{n}^{\prime}, \boldsymbol{n}_{1}=\boldsymbol{n}$, and the condition (33) becomes trivial: $\sigma_{1}-\sigma_{2}-\sigma=0$.

The general case corresponds to the partial wetting $(\sin \theta \neq 0)$. Due to (18),

$$
\boldsymbol{n}^{\prime T} \frac{\partial \sigma}{\partial \boldsymbol{R}} \boldsymbol{P} \equiv \boldsymbol{n}^{\prime T}(a \boldsymbol{I}+b \boldsymbol{R}) \boldsymbol{P} \equiv a \boldsymbol{n}^{\prime T}+b \boldsymbol{n}^{\prime T} \boldsymbol{R} \equiv \boldsymbol{n}^{\prime T} \frac{\partial \sigma}{\partial \boldsymbol{R}} .
$$

Hence, (32) yields

$$
\boldsymbol{n}^{\prime T} \frac{\partial \sigma}{\partial \boldsymbol{R}}=0
$$

Equation (34) implies (see Lemma 4)

$$
\boldsymbol{n}^{\prime T}\left[\left(\frac{\partial \sigma}{\partial H}+H \frac{\partial \sigma}{\partial K}\right) \boldsymbol{I}-\frac{\partial \sigma}{\partial K} \boldsymbol{R}\right]=0 .
$$

Consequently, $\boldsymbol{n}^{\prime}$ is an eigenvector of $\boldsymbol{R}$. We denote by $c_{n^{\prime}}$ the associated eigenvalue $c_{2}$. Then

$$
\frac{\partial \sigma}{\partial H}+H \frac{\partial \sigma}{\partial K}=c_{n^{\prime}} \frac{\partial \sigma}{\partial K} .
$$

Due to the fact that $\boldsymbol{t}$ is also an eigenvector of $\boldsymbol{R}$ with eigenvalue $c_{t}=c_{1}\left(\boldsymbol{t}\right.$ and $\boldsymbol{n}^{\prime}$ form the eigenbasis of $\boldsymbol{R}$ along $C_{t}$ ), we get $H=c_{t}+c_{n^{\prime}}$ and the equivalent to the boundary condition (35) in the form

$$
\frac{\partial \sigma}{\partial H}+c_{t} \frac{\partial \sigma}{\partial K}=0
$$

From Lemma 4, (16), we immediately deduce

$$
\operatorname{div}_{\operatorname{tg}}\left(\boldsymbol{P} \frac{\partial \sigma}{\partial \boldsymbol{R}}\right)=\nabla_{\mathrm{tg}}^{T} a+\left(a H+b H^{2}-2 b K\right) \boldsymbol{n}^{T}+\nabla_{\operatorname{tg}}^{T} b \boldsymbol{R}+b \nabla_{\mathrm{tg}}^{T} H .
$$


Due to the fact that $\boldsymbol{n}^{\prime T} \boldsymbol{n}=0$, we obtain

$$
\boldsymbol{n}^{\prime T} \operatorname{div}_{\operatorname{tg}}^{T}\left(\boldsymbol{P} \frac{\partial \sigma}{\partial \boldsymbol{R}}\right)=\boldsymbol{n}^{\prime T}\left[\nabla_{\mathrm{tg}} a+\boldsymbol{R} \nabla_{\mathrm{tg}} b+b \nabla_{\mathrm{tg}} H\right]=\boldsymbol{n}^{\prime T}[\nabla a+\boldsymbol{R} \nabla b+b \nabla H] .
$$

Consequently, one obtains the second condition on $C_{t}$ in the form

$$
\sigma_{1}-\sigma_{2}+\sigma \cos \theta-\sin \theta \boldsymbol{n}^{\prime T}(\nabla a+b \nabla H+\boldsymbol{R} \nabla b)=0 .
$$

This is the extended Young-Dupré condition along contact line $C_{t}$ between membrane $S_{t}$ and solid surface $\mathscr{S}^{3}$

In the case of Helfrich's energy given by relation (1), we obtain the extended Young-Dupré condition (38) in the form:

$$
\sigma_{1}-\sigma_{2}+\sigma \cos \theta-\kappa \sin \theta \boldsymbol{n}^{\prime T} \nabla H=0 .
$$

This last condition was previously obtained in [Gouin 2014b].

\section{Surfaces of revolution}

8.1. Shape equation for the surfaces of revolution. Along a revolution surface, the invariants of the curvature tensor depend only on $s$, which is the curvilinear abscissa of meridian curve denoted by $\Gamma$ [Aleksandrov and Zalgaller 1967]:

$$
H=H(s), \quad K=K(s) .
$$

${ }^{3}$ The virtual displacement taken in the most general form (28) does not produce new boundary conditions. Due to the linearity of the virtual work, to prove this property it is sufficient to take $\zeta=\alpha \boldsymbol{t}$. We obtain

$$
\frac{\partial \zeta}{\partial \boldsymbol{x}}=\boldsymbol{t}(\nabla \alpha)^{T}+\alpha \frac{\partial \boldsymbol{t}}{\partial \boldsymbol{x}}, \quad\left(\frac{\partial \zeta}{\partial \boldsymbol{x}}\right)^{T} \boldsymbol{n}_{1}^{\prime}=\alpha\left(\frac{\partial \boldsymbol{t}}{\partial \boldsymbol{x}}\right)^{T} \boldsymbol{n}_{1}^{\prime}
$$

Since

$$
\frac{\partial t}{\partial \boldsymbol{x}}=c \boldsymbol{N} \boldsymbol{t}^{T},
$$

where $N$ is the principal unit normal and $c$ is the curvature along $C_{t}$, one obtains

$$
\left(\frac{\partial \zeta}{\partial \boldsymbol{x}}\right)^{T} \boldsymbol{n}_{1}^{\prime}=\alpha c \boldsymbol{t} \boldsymbol{N}^{T} \boldsymbol{n}_{1}^{\prime}
$$

and

$$
\sin \theta \boldsymbol{n}^{\prime T} \frac{\partial \sigma}{\partial \boldsymbol{R}} \boldsymbol{P}\left(\frac{\partial \zeta}{\partial \boldsymbol{x}}\right)^{T} \boldsymbol{n}_{1}^{\prime}=\alpha c \sin \theta \boldsymbol{n}^{\prime T} \frac{\partial \sigma}{\partial \boldsymbol{R}} \boldsymbol{t} \boldsymbol{N}^{T} \boldsymbol{n}_{1}^{\prime}
$$

which is equal to zero thanks to (34).

Moreover, thanks to (34), we immediately obtain that term

$$
\left[\left(\sigma_{1}-\sigma_{2}\right) \boldsymbol{n}_{1}^{\prime T}+\sigma \boldsymbol{n}^{\prime T}-\boldsymbol{n}^{\prime T} \operatorname{div}_{\operatorname{tg}}^{T}\left(\boldsymbol{P} \frac{\partial \sigma}{\partial \boldsymbol{R}}\right) \boldsymbol{n}^{T}-\boldsymbol{n}^{\prime T} \frac{\partial \sigma}{\partial \boldsymbol{R}} \boldsymbol{R}+\cos \theta \boldsymbol{n}^{\prime T} \frac{\partial \sigma}{\partial \boldsymbol{R}} \boldsymbol{P}\left(\frac{\partial \boldsymbol{n}_{1}}{\partial \boldsymbol{x}}\right)^{T}\right] \boldsymbol{t} \alpha
$$

is vanishing. Hence, new boundary conditions do not appear on $C_{t}$. 


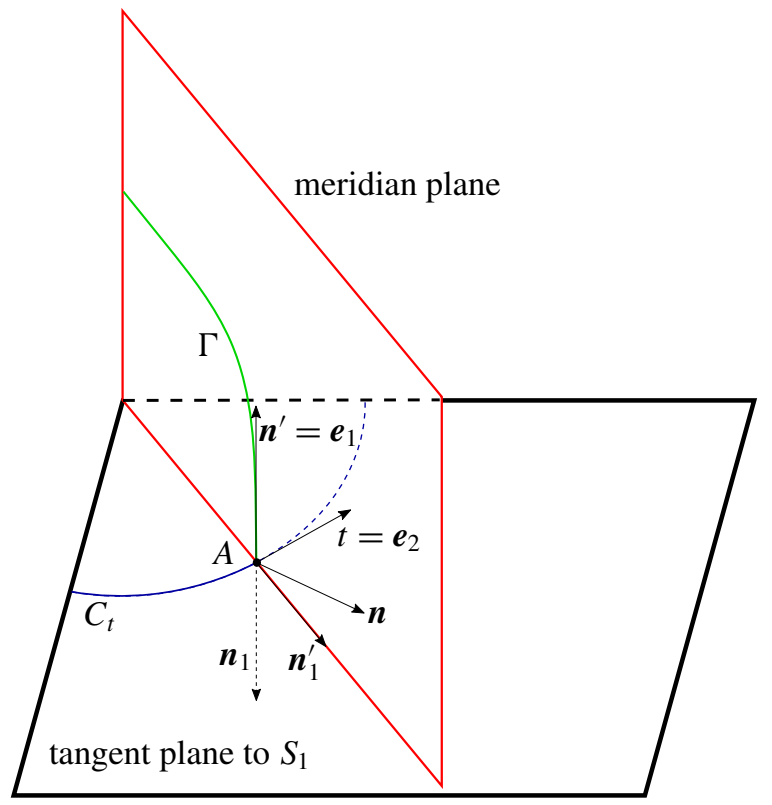

Figure 3. The case of a revolution domain. The line $C_{t}$ (contact edge between $S_{t}$ and $S_{1}$ ) is a circle with an axis which is the revolution axis collinear to $\boldsymbol{n}_{1}$. The meridian curve is denoted $\Gamma$; normal vector $\boldsymbol{n}$ and binormal vector $\boldsymbol{n}^{\prime}$ are in the meridian plane of revolution surface $S_{t}$. We have $\boldsymbol{n}^{\prime}=\boldsymbol{e}_{1}$ and $\boldsymbol{t}=\boldsymbol{e}_{2}$, corresponding to the eigenvectors of the curvature tensor $\boldsymbol{R}$ at $A$.

One of the eigenvectors, denoted $\boldsymbol{e}_{1}$, of the curvature tensor $\boldsymbol{R}$ is tangent to meridian curve $\Gamma$ (see Figure 3). Let us remark that for any function $f(s)$, one has

$$
\nabla_{\operatorname{tg}} f=\frac{d f}{d s} \boldsymbol{e}_{1}, \quad \Delta_{\mathrm{tg}} f=\frac{d^{2} f}{d s^{2}} .
$$

Indeed, the first equation is the definition of the tangential gradient. The second equality is obtained as follows:

$$
\begin{aligned}
\operatorname{div}_{\operatorname{tg}}\left(\frac{d f}{d s} \boldsymbol{e}_{1}\right)=\operatorname{tr}\left(\boldsymbol{P} \frac{\partial}{\partial \boldsymbol{x}}\left(\frac{d f}{d s} \boldsymbol{e}_{1}\right)\right) & =\operatorname{tr}\left(\boldsymbol{P} \frac{d}{d s}\left(\frac{d f}{d s} \boldsymbol{e}_{1}\right) \otimes \boldsymbol{e}_{1}\right) \\
& =\operatorname{tr}\left(\frac{d^{2} f}{d s^{2}} \boldsymbol{P} \boldsymbol{e}_{1} \otimes \boldsymbol{e}_{1}+c_{1}(s) \frac{d f}{d s} \boldsymbol{n} \otimes \boldsymbol{e}_{1}\right)=\frac{d^{2} f}{d s^{2}} .
\end{aligned}
$$

The Frénet formula was used here:

$$
\frac{d \boldsymbol{e}_{1}}{d s}=c_{1} \boldsymbol{n}
$$


Also,

$$
\operatorname{div}_{\operatorname{tg}}\left(\boldsymbol{R} \nabla_{\operatorname{tg}} f\right)=\operatorname{div}_{\operatorname{tg}}\left(\frac{d f}{d s} \boldsymbol{R e}_{1}\right)=\operatorname{div}_{\operatorname{tg}}\left(\frac{d f}{d s} c_{1} \boldsymbol{e}_{1}\right)=\frac{d}{d s}\left(c_{1} \frac{d f}{d s}\right) .
$$

For surfaces of revolution the shape equation (26) becomes

$$
\begin{aligned}
H\left(\sigma-K \frac{\partial \sigma}{\partial K}\right)+\left(2 K-H^{2}\right) \frac{\partial \sigma}{\partial H} & -\frac{d^{2}}{d s^{2}}\left(\frac{\partial \sigma}{\partial H}\right)-H \frac{d^{2}}{d s^{2}}\left(\frac{\partial \sigma}{\partial K}\right) \\
& -\frac{d H}{d s} \frac{d}{d s}\left(\frac{\partial \sigma}{\partial K}\right)+\frac{d}{d s}\left(c_{1} \frac{d}{d s}\left(\frac{\partial \sigma}{\partial K}\right)\right)=\mathscr{P}-p .
\end{aligned}
$$

8.2. Extended Young-Dupré condition for surfaces of revolution. One has along $C_{t}, \boldsymbol{t}=\boldsymbol{e}_{2}$ and $\boldsymbol{n}^{\prime}=\boldsymbol{e}_{1}$. It implies $\boldsymbol{n}^{\prime T} \boldsymbol{R} \boldsymbol{t}=0$. Also, one has

$$
\boldsymbol{n}^{\prime T}(\nabla a+b \nabla H+\boldsymbol{R} \nabla b)=\frac{d a}{d s}+b \frac{d H}{d s}+c_{1} \frac{d b}{d s} .
$$

The Young-Dupré condition (38) becomes

$$
\sigma_{1}-\sigma_{2} \cos \theta-\sin \theta\left(\frac{d a}{d s}+b \frac{d H}{d s}+c_{t} \frac{d b}{d s}\right)=0 .
$$

Since

$$
a=\frac{\partial \sigma}{\partial H}+H \frac{\partial \sigma}{\partial K}, \quad b=-\frac{\partial \sigma}{\partial K},
$$

one finally obtains

$$
\sigma_{1}-\sigma_{2} \cos \theta-\sin \theta\left[\frac{d}{d s}\left(\frac{\partial \sigma}{\partial H}\right)+c_{n^{\prime}} \frac{d}{d s}\left(\frac{\partial \sigma}{\partial K}\right)\right]=0 .
$$

For the Helfrich energy (1) this expression yields

$$
\sigma_{1}-\sigma_{2} \cos \theta-\kappa \frac{d H}{d s} \sin \theta=0 .
$$

\section{Conclusion}

Membranes can be considered as material surfaces endowed with a surface energy density depending on the invariants of the curvature tensor: $\sigma=\sigma(H, K)$. By using the principle of virtual working, we derived the boundary conditions on the moving membranes ("shape equation") as well as two boundary conditions on the contact line. In limit cases, we recover classical boundary conditions. The "shape equation" and the boundary conditions are summarized below in the nondegenerate case (see (26), (36), and (38)) as the following: 
- The equation for the moving surface $S_{t}$ :

$$
\begin{aligned}
H\left(\sigma-K \frac{\partial \sigma}{\partial K}\right)+\left(2 K-H^{2}\right) \frac{\partial \sigma}{\partial H} & -\Delta_{\operatorname{tg}} \frac{\partial \sigma}{\partial H}-H \Delta_{\operatorname{tg}} \frac{\partial \sigma}{\partial K} \\
& -\nabla_{\operatorname{tg}}^{T} H \nabla_{\operatorname{tg}} \frac{\partial \sigma}{\partial K}+\operatorname{div}_{\operatorname{tg}}\left(\boldsymbol{R} \nabla_{\operatorname{tg}} \frac{\partial \sigma}{\partial K}\right)=\mathscr{P}-p .
\end{aligned}
$$

- The clamping condition on the moving line $C_{t}$ :

$$
\frac{\partial \sigma}{\partial H}+c_{t} \frac{\partial \sigma}{\partial K}=0 .
$$

Also, $\left(\boldsymbol{t}, \boldsymbol{n}, \boldsymbol{n}^{\prime}\right)$ - which is the Darboux frame — are the eigenvectors of curvature tensor $\boldsymbol{R}$.

- Dynamic generalization of the Young-Dupré condition on $C_{t}$ :

$$
\sigma_{1}-\sigma_{2}+\sigma \cos \theta-\sin \theta \boldsymbol{n}^{\prime T}\left(\nabla_{\operatorname{tg}}\left(\frac{\partial \sigma}{\partial H}\right)+(H \boldsymbol{P}-\boldsymbol{R}) \nabla_{\operatorname{tg}}\left(\frac{\partial \sigma}{\partial K}\right)\right)=0 .
$$

In the case of Helfrich's energy the generalization of the Young-Dupré condition is reduced to (39):

$$
\sigma_{1}-\sigma_{2}+\sigma \cos \theta-\kappa \sin \theta \boldsymbol{n}^{\prime T} \nabla_{\operatorname{tg}} H=0 .
$$

The last term, corresponding to the variation of the mean curvature of $S_{t}$ in the binormal direction at the contact line, can dominate the other terms. It could be interpreted as a line tension term usually added in the models with constant surface energy [Babak 2004]. It should also be noted that the droplet volume has no effect in the classical Young-Dupré condition. This is not the case for the generalized Young-Dupré condition since the curvatures can become very large for very small droplets (they are inversely proportional to the droplet size). The clamping condition for the Helfrich energy fixes the value of $H$ on the contact line:

$$
H=H_{0}-c_{t} \frac{\bar{\kappa}}{\kappa} \text {. }
$$

The new shape equation and boundary conditions can be used for solving dynamic problems. This could be, for example, the study of the "fingering" phenomenon appearing as a result of the nonlinear instability of a moving contact line. This complicated problem will be studied in the future.

\section{Appendix}

Since $\sigma=\sigma(H, K)$, we get

$$
\nabla_{\mathrm{tg}} \sigma=\frac{\partial \sigma}{\partial H} \nabla_{\operatorname{tg}} H+\frac{\partial \sigma}{\partial K} \nabla_{\mathrm{tg}} K
$$


From (16), we obtain

$$
\operatorname{div}_{\operatorname{tg}}\left(\boldsymbol{P} \frac{\partial \sigma}{\partial \boldsymbol{R}}\right)=\nabla_{\mathrm{tg}}^{T} a+\left(a H+b H^{2}-2 b K\right) \boldsymbol{n}^{T}+\nabla_{\mathrm{tg}}^{T} b \boldsymbol{R}+b \nabla_{\mathrm{tg}}^{T} H .
$$

Also, one has

$$
\operatorname{div}_{\mathrm{tg}}\left(\frac{\partial \sigma}{\partial \boldsymbol{R}} \boldsymbol{R}\right)=\operatorname{div}_{\mathrm{tg}}(a \boldsymbol{R})+\operatorname{div}_{\mathrm{tg}}\left(b \boldsymbol{R}^{2}\right)
$$

Due to (9), one has

$$
\begin{aligned}
\operatorname{div}_{\operatorname{tg}}(a \boldsymbol{R}) & =\left(\nabla_{\mathrm{tg}}^{T} a\right) \boldsymbol{R}+a \nabla_{\mathrm{tg}}^{T} H+a\left(H^{2}-2 K\right) \boldsymbol{n}^{T}, \\
\operatorname{div}_{\mathrm{tg}}\left(b \boldsymbol{R}^{2}\right) & =\operatorname{div}_{\mathrm{tg}}[b(H \boldsymbol{R}-K \boldsymbol{P})] \\
& =\nabla_{\mathrm{tg}}^{T}(b H) \boldsymbol{R}+b H\left[\nabla_{\mathrm{tg}}^{T} H+\left(H^{2}-2 K\right) \boldsymbol{n}^{T}\right]-\nabla_{\mathrm{tg}}^{T}(b K)-b K H \boldsymbol{n}^{T} .
\end{aligned}
$$

Consequently,

$$
\begin{aligned}
\operatorname{div}_{\mathrm{tg}}\left(\frac{\partial \sigma}{\partial \boldsymbol{R}} \boldsymbol{R}\right) & =\left(\nabla_{\mathrm{tg}}^{T}(a+b H)\right) \boldsymbol{R} \\
& +(a+b H) \nabla_{\mathrm{tg}}^{T} H-\nabla_{\mathrm{tg}}^{T}(b K)+\left(a H^{2}+b H^{3}-2 a K-3 b H K\right) \boldsymbol{n}^{T} .
\end{aligned}
$$

From relations (40), (41), and (42), we deduce

$$
\nabla_{\mathrm{tg}} \sigma-\operatorname{div}_{\mathrm{tg}}^{T}\left(\frac{\partial \sigma}{\partial \boldsymbol{R}} \boldsymbol{R}\right)+\boldsymbol{R} \operatorname{div}_{\operatorname{tg}}^{T}\left(\boldsymbol{P} \frac{\partial \sigma}{\partial \boldsymbol{R}}\right)=\left(2 a K+3 b H K-a H^{2}-b H^{3}\right) \boldsymbol{n} .
$$

Using (41), one obtains

$$
\boldsymbol{P} \operatorname{div}_{\mathrm{tg}}^{T}\left(\boldsymbol{P} \frac{\partial \sigma}{\partial \boldsymbol{R}}\right)=\nabla_{\mathrm{tg}} a+\boldsymbol{R} \nabla_{\mathrm{tg}} b+b \nabla_{\mathrm{tg}} H
$$

One deduces

$$
\operatorname{div}_{\mathrm{tg}}\left[\boldsymbol{P} \operatorname{div}_{\mathrm{tg}}^{T}\left(\boldsymbol{P} \frac{\partial \sigma}{\partial \boldsymbol{R}}\right)\right]=\Delta_{\mathrm{tg}} a+\operatorname{div}_{\mathrm{tg}}\left(\boldsymbol{R} \nabla_{\mathrm{tg}} b\right)+b \Delta_{\mathrm{tg}} H+\nabla_{\mathrm{tg}}^{T} b \nabla_{\mathrm{tg}} H .
$$

From relations (40), (41), and (42), we deduce

$$
\begin{aligned}
\nabla_{\mathrm{tg}} \sigma-\operatorname{div}_{\mathrm{tg}}^{T}\left(\frac{\partial \sigma}{\partial \boldsymbol{R}} \boldsymbol{R}\right)+ & \boldsymbol{R} \operatorname{div}_{\operatorname{tg}}^{T}\left(\boldsymbol{P} \frac{\partial \sigma}{\partial \boldsymbol{R}}\right) \\
=(2 a K+3 b & \left.H K-a H^{2}-b H^{3}\right) \boldsymbol{n}+\frac{\partial \sigma}{\partial H} \nabla_{\mathrm{tg}} H+\frac{\partial \sigma}{\partial K} \nabla_{\mathrm{tg}} K \\
- & \boldsymbol{R} \nabla_{\mathrm{tg}}(a+b H)-(a+b H) \nabla_{\mathrm{tg}} H+\nabla_{\mathrm{tg}}(b K)+\boldsymbol{R} \nabla_{\mathrm{tg}} a \\
+ & \left(a H+b H^{2}-2 b K\right) \boldsymbol{R} \boldsymbol{n}+\boldsymbol{R}^{2} \nabla_{\mathrm{tg}} b+b \boldsymbol{R} \nabla_{\mathrm{tg}} H+\boldsymbol{T}
\end{aligned}
$$$$
=\mathbf{0} \text {. }
$$ 
Using relations $\boldsymbol{R} \boldsymbol{n}=\mathbf{0}$, Lemma 1 (identity 3) and expressions of $a$ and $b$ given by (16), we obtain

$$
\begin{array}{rl}
\frac{\partial \sigma}{\partial H} \nabla_{\mathrm{tg}} H+\frac{\partial \sigma}{\partial K} \nabla_{\mathrm{tg}} & K-\boldsymbol{R} \nabla_{\mathrm{tg}}(a+b H)-(a+b H) \nabla_{\mathrm{tg}} H+\nabla_{\mathrm{tg}}(b K) \\
& +\boldsymbol{R} \nabla_{\mathrm{tg}} a+\left(a H+b H^{2}-2 b K\right) \boldsymbol{R} \boldsymbol{n}+\boldsymbol{R}^{2} \nabla_{\mathrm{tg}} b+b \boldsymbol{R} \nabla_{\mathrm{tg}} H=\mathbf{0} .
\end{array}
$$

Consequently,

$$
\nabla_{\mathrm{tg}} \sigma-\operatorname{div}_{\mathrm{tg}}^{T}\left(\frac{\partial \sigma}{\partial \boldsymbol{R}} \boldsymbol{R}\right)+\boldsymbol{R} \operatorname{div}_{\mathrm{tg}}^{T}\left(\boldsymbol{P} \frac{\partial \sigma}{\partial \boldsymbol{R}}\right)=\left(2 a K+3 b H K-a H^{2}-b H^{3}\right) \boldsymbol{n} .
$$

Finally, using (43), one obtains

$$
\begin{aligned}
{\left[p+H \sigma-\Delta_{\mathrm{tg}} a-b \Delta_{\mathrm{tg}} H-\right.} & \nabla_{\mathrm{tg}}^{T} b \nabla_{\mathrm{tg}} H-\operatorname{div}_{\mathrm{tg}}\left(\boldsymbol{R} \nabla_{\mathrm{tg}} b\right) \\
& \left.+\left(2 a K+3 b H K-a H^{2}-b H^{3}\right)\right] \boldsymbol{n}+\boldsymbol{T}=\mathbf{0},
\end{aligned}
$$

where all tangential terms disappear in the boundary condition on $S_{t}$.

\section{Acknowledgments}

The authors thank the anonymous referees for their noteworthy and helpful remarks that greatly contributed to improve the final version of the paper.

\section{References}

[Alberts et al. 2002] B. Alberts, A. Johnson, J. Lewis, M. Raff, K. Roberts, and P. Walter, Molecular biology of the cell, 4th ed., Garland Science, New York, 2002.

[Aleksandrov and Zalgaller 1967] A. D. Aleksandrov and V. A. Zalgaller, Intrinsic geometry of surfaces, Translations of Mathematical Monographs 15, American Mathematical Society, Providence, R.I., 1967.

[Babak 2004] V. G. Babak, "Stability of the lenslike liquid thickening (the drop) on a solid substrate", J. Adhesion 80:8 (2004), 685-703.

[Berdichevsky 2009] V. L. Berdichevsky, Variational principles of continuum mechanics, I: Fundamentals, Springer, 2009.

[Biscari et al. 2004] P. Biscari, S. M. Canevese, and G. Napoli, "Impermeability effects in threedimensional vesicles", J. Phys. A 37:27 (2004), 6859-6874.

[Capovilla and Guven 2002] R. Capovilla and J. Guven, "Stresses in lipid membranes", J. Phys. A 35:30 (2002), 6233-6247.

[Fournier 2007] J.-B. Fournier, "On the stress and torque tensors in fluid membranes", Soft Matter 3 (2007), 883-888.

[Gavrilyuk 2011] S. Gavrilyuk, "Multiphase flow modeling via Hamilton's principle”, pp. 163-210 in Variational models and methods in solid and fluid mechanics, edited by F. dell'Isola and S. Gavrilyuk, CISM Courses and Lect. 535, Springer, 2011.

[Gavrilyuk and Gouin 1999] S. Gavrilyuk and H. Gouin, "A new form of governing equations of fluids arising from Hamilton's principle”, Internat. J. Engrg. Sci. 37:12 (1999), 1495-1520. 
[Germain 1973] P. Germain, "The method of virtual power in continuum mechanics, 2: Microstructure", SIAM J. Appl. Math. 25:3 (1973), 556-575.

[Gouin 2007] H. Gouin, "The d'Alembert-Lagrange principle for gradient theories and boundary conditions", pp. 79-95 in Asymptotic methods in nonlinear wave phenomena, edited by T. Ruggeri and M. Sammartino, World Scientific, 2007.

[Gouin 2014a] H. Gouin, "Interfaces endowed with nonconstant surface energies revisited with the d'Alembert-Lagrange principle", Math. Mech. Complex Syst. 2:1 (2014), 23-43.

[Gouin 2014b] H. Gouin, "Vesicle model with bending energy revisited", Acta Appl. Math. 132 (2014), 347-358.

[Helfrich 1973] W. Helfrich, "Elastic properties of lipid bilayers: theory and possible experiments", Z. Naturforsch. C. 28:11-12 (1973), 693-703.

[Lipowsky and Sackmann 1995] R. Lipowsky and E. Sackmann (editors), Structure and dynamics of membranes: from cells to vesicles, Handbook of Biological Physics 1, Elsevier, Amsterdam, 1995.

[Napoli and Vergori 2010] G. Napoli and L. Vergori, "Equilibrium of nematic vesicles", J. Phys. A 43:44 (2010), 445207.

[Rocard 1952] Y. Rocard, Thermodynamique, Masson, Paris, 1952.

[Rosso and Virga 1999] R. Rosso and E. G. Virga, "Adhesive borders of lipid membranes", Proc. Roy. Soc. Lond. A 455:1992 (1999), 4145-4168.

[Schwartz 1966] L. Schwartz, Théorie des distributions, Publications de l'Institut de Mathématique de l’Université de Strasbourg 9-10, Hermann, 1966.

[Seifert 1997] U. Seifert, "Configurations of fluid membranes and vesicles", Adv. Phys. 46:1 (1997), 13-137.

[Serrin 1959] J. Serrin, "Mathematical principles of classical fluid mechanics”, pp. 125-263 in Handbuch der Physik, vol. 8/1: Strömungsmechanik I, edited by S. Flügge, Springer, 1959.

[Steigmann and Li 1995] D. J. Steigmann and D. Li, "Energy-minimizing states of capillary systems with bulk, surface, and line phases", IMA J. Appl. Math. 55:1 (1995), 1-17.

[Tu 2011] Z. Tu, "Geometry of membranes", J. Geom. Symmetry Phys. 24 (2011), 45-75.

[Willmore 1993] T. J. Willmore, Riemannian geometry, Clarendon, New York, 1993.

[Zhong-can and Helfrich 1989] O.-Y. Zhong-can and W. Helfrich, "Bending energy of vesicle membranes: general expressions for the first, second, and third variation of the shape energy and applications to spheres and cylinders", Phys. Rev. A 39:10 (1989), 5280-5288.

Received 12 Oct 2018. Revised 20 Feb 2019. Accepted 2 Apr 2019.

SERGEY GAVRILYUK: sergey.gavrilyuk@univ-amu.fr

Aix Marseille Univ, CNRS, IUSTI, UMR 7343, Marseille, France

HENRI GOUIN: henri.gouin@univ-amu.fr, henri.gouin@yahoo.fr

Aix Marseille Univ, CNRS, IUSTI, UMR 7343, Marseille, France 
EDITORIAL BOARD

ANTONIO CARCATERRA

ERIC A. CARLEN

FRANCESCO DELL'ISOLA

RAFFAELE ESPOSITO

ALBERT FANNJIANG

Gilles A. FrancFort

Pierangelo Marcati

JEAN-JACQUES MARIGO

PETER A. MARKOWICH

MARTIN OSTOJA-STARZEWSKI

PIERRE SEPPECHER

DAVID J. STEIGMANN

PAUL STEINMANN

PierRe M. SuQueT

MANAGING EDITORS

MICOL AMAR

ANGELA MADEO

MARTIN OSTOJA-STARZEWSKI

\section{ADVISORY BOARD}

ADNAN AKAY

Holm AltenbaCH

MICOL AMAR

HARM ASKES

TEODOR ATANACKOVIĆ

VICTOR BERDICHEVSKY

GuY BouchitTÉ

ANDREA BRAIDES

ROBERTO CAMASSA

MAURO CARFORE

ERIC DARVE

FELIX DARVE

ANNA DE MASI

Gianpietro Del Piero

Emmanuele Di Benedetto

VICTOR A. EREMEYEV

BERNOLD FIEDLER

IRENE M. GAMBA

DAVID Y. GAO

SERGEy GaVRILYUK

Timothy J. HeAley

DOMINIQUE JEULIN

ROgER E. KHAYAT

CORRADO LATTANZIO

ROBERT P. LIPTON

ANGELO LUONGO

ANGEla MADEO

JUAN J. MANFREDI

CARlo MARCHIORO

ANIL MISRA

ROBERTO NATALINI

PATRIZIO NEFF

Thomas J. Pence

ANDREY PIATNITSKI

ERRICO PRESUTtI

MARIO PUlVIRENTI

Lucio Russo

Miguel A. F. SANJUAN

PATRICK SElvaduraI

MIROSLAV ŠILHAVÝ

GUIDO SWEERS

ANTOINETTE TORDESILLAS

LEV TRUSKINOVSKY

JUAN J. L. VELÁZQUEZ

VINCENZO VESPRI

ANGELO VULPIANI msp.org/memocs

Università di Roma "La Sapienza", Italia

Rutgers University, USA

(CO-CHAIR) Università di Roma "La Sapienza", Italia

(TREASURER) Università dell'Aquila, Italia

University of California at Davis, USA

(CO-CHAIR) Université Paris-Nord, France

Università dell' Aquila, Italy

École Polytechnique, France

DAMTP Cambridge, UK, and University of Vienna, Austria

(CHAIR MANAGING EDITOR) Univ. of Illinois at Urbana-Champaign, USA

Université du Sud Toulon-Var, France

University of California at Berkeley, USA

Universität Erlangen-Nürnberg, Germany

LMA CNRS Marseille, France

Università di Roma "La Sapienza", Italia

Université de Lyon-INSA (Institut National des Sciences Appliquées), France (CHAIR MANAGING EDITOR) Univ. of Illinois at Urbana-Champaign, USA

Carnegie Mellon University, USA, and Bilkent University, Turkey

Otto-von-Guericke-Universität Magdeburg, Germany

Università di Roma "La Sapienza”, Italia

University of Sheffield, UK

University of Novi Sad, Serbia

Wayne State University, USA

Université du Sud Toulon-Var, France

Università di Roma Tor Vergata, Italia

University of North Carolina at Chapel Hill, USA

Università di Pavia, Italia

Stanford University, USA

Institut Polytechnique de Grenoble, France

Università dell' Aquila, Italia

Università di Ferrara and International Research Center MEMOCS, Italia

Vanderbilt University, USA

Gdansk University of Technology, Poland

Freie Universität Berlin, Germany

University of Texas at Austin, USA

Federation University and Australian National University, Australia

Université Aix-Marseille, France

Cornell University, USA

École des Mines, France

University of Western Ontario, Canada

Università dell' Aquila, Italy

Louisiana State University, USA

Università dell'Aquila, Italia

Université de Lyon-INSA (Institut National des Sciences Appliquées), France University of Pittsburgh, USA

Università di Roma "La Sapienza", Italia

University of Kansas, USA

Istituto per le Applicazioni del Calcolo "M. Picone", Italy

Universität Duisburg-Essen, Germany

Michigan State University, USA

Narvik University College, Norway, Russia

Università di Roma Tor Vergata, Italy

Università di Roma “La Sapienza”, Italia

Università di Roma “Tor Vergata", Italia

Universidad Rey Juan Carlos, Madrid, Spain

McGill University, Canada

Academy of Sciences of the Czech Republic

Universität zu Köln, Germany

University of Melbourne, Australia

École Polytechnique, France

Bonn University, Germany

Università di Firenze, Italia

Università di Roma La Sapienza, Italia

MEMOCS (ISSN 2325-3444 electronic, 2326-7186 printed) is a journal of the International Research Center for the Mathematics and Mechanics of Complex Systems at the Università dell'Aquila, Italy.

Cover image: "Tangle” by $\odot$ John Horigan; produced using the Context Free program (contextfreeart.org).

PUBLISHED BY

7 mathematical sciences publishers

nonprofit scientific publishing

http://msp.org/

(C) 2019 Mathematical Sciences Publishers 
Mathematics and Mechanics of Complex Systems vol. 7 no. 2

A polynomial chaos expanded hybrid fuzzy-stochastic model for transversely fiber reinforced plastics

Eduard Penner, Ismail Caylak, Alex Dridger and Rolf Mahnken

Dynamic boundary conditions for membranes whose surface energy depends on the mean and Gaussian curvatures

Sergey Gavrilyuk and Henri Gouin Energy-based trajectory tracking and vibration control for multilink highly flexible manipulators

Ivan Giorgio and Dionisio Del Vescovo

A model of the proppant flowback: setup of the theoretical framework

Ksenia P. Frolova, Polina M. Grigoreva, Konstantin E.

Lezhnev and Grigoriy V. Paderin

The object detection by autonomous apparatus as a solution of the 189 Buffon needle problem

Mikhail A. Guzev, Gurami S. Tsitsiashvili and Marina A.

Osipova

MEMOCS is a journal of the International Research Center for the Mathematics and Mechanics of Complex Systems at the Università dell' Aquila, Italy.

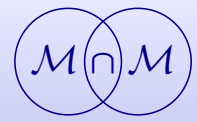

